\title{
Longitudinal holes in debunched particle beams in storage rings, perpetuated by space-charge forces
}

\author{
Shane Koscielniak \\ TRIUMF, 4004 Wesbrook Mall, Vancouver, British Columbia V6T 2A3, Canada \\ Steven Hancock and Mats Lindroos \\ CERN, CH-1211, Geneva 23, Switzerland \\ (Received 5 October 2000; published 2 April 2001)
}

\begin{abstract}
Stationary, self-consistent, and localized longitudinal density perturbations on an unbunched chargedparticle beam, which are solutions of the nonlinearized Vlasov-Poisson equation, have recently received some attention. In particular, we address the case that space charge is the dominant longitudinal impedance and the storage ring operates below transition energy so that the negative mass instability is not an explanation for persistent beam structure. Under the customary assumption of a bell-shaped steadystate distribution, about which the expansion is made, the usual wave theory of Keil and Schnell for perturbations on unbunched beams predicts that self-sustaining perturbations are possible only (below transition) if the impedance is inductive (or resistive) or if the bell shape is inverted. Space charge gives a capacitive impedance. Nevertheless, we report numerous experimental measurements made at the CERN Proton Synchrotron Booster that plainly show the longevity of holelike structures in coasting beams. We shall also report on computer simulations of boosterlike beams that provide compelling evidence that it is space-charge force which perpetuates the holes. We shall show that the localized solitonlike structures, i.e., holes, decouple from the steady-state distribution and that they are simple solutions of the nonlinearized time-independent Vlasov equation. We have derived conditions for stationarity of holes that satisfy the requirement of self-consistency; essentially, the relation between the momentum spread and depth of the holes is given by the Hamiltonian — with the constraint that the phase-space density be high enough to support the solitons. The stationarity conditions have scaling laws similar to the Keil-Schnell criteria except that the charge and momentum spread of the hole replaces that of the beam.
\end{abstract}

DOI: 10.1103/PhysRevSTAB.4.044201

PACS numbers: 29.20.Lq, 29.27.Bd, 52.35.Sb

\section{INTRODUCTION}

Stationary, self-consistent, and localized longitudinal density perturbations on an unbunched charged-particle beam, which are solutions of the nonlinearized VlasovPoisson equation, have recently received some attention in the literature. In particular, we shall address the case that "space charge" is the dominant longitudinal impedance and the storage ring operates below transition energy so that the negative mass instability is not an explanation for persistent beam structure.

Colestock [1] and Spentzouris [2] have reported some experimental observations, and we shall report on numerous measurements [3-5] made at the CERN Proton Synchrotron Booster (PSB). In particular, Ref. [1] reports the observation of notches in beam transfer function [6] measurements; such features were also observed by Sacherer [7] many years ago in the PSB.

Some theoretical investigations have been made by Schamel [8-11] who claims that the perturbations are solitonlike and cannot be predicted by the linearized Vlasov treatment used by Keil and Schnell [12]; we agree with this claim, and explain why. Reference [9] studies a pure resistive wake, while Ref. [11] considers a reactive wake. The existence of solitary waves, wherein dispersion is balanced against nonlinearity, has also been proposed by Bisognano [13] and was discussed by Tzenov [14].

The customary [15] space-charge impedance has no "roll off" at higher frequency and is formally equivalent to a pure capacitive impedance. The space-charge forces within a local rarefaction of the particle density are focusing toward the center of the perturbation, and so, intuitively, one might expect the possibility that holes in beams below transition energy could be self-stabilized.

In terms of the usual wave theory $[12,16,17]$ for perturbations on unbunched beams, in order to construct a localized, stationary distribution one must be able to superpose a system of standing waves of the form $e^{i\left(n x-\omega_{n} t\right)}$. For the impedance due to space charge and the induced charges in a perfectly conducting wall, the constancy of impedance divided by wave number $Z\left(n \omega_{\text {rev }}\right) / n$ implies the eigenfrequencies $\omega_{n}=n \omega_{1}$ and that all waves have the same speed; consequently, one might expect to be able to construct localized, stationary perturbations. This hypothesis was put forward by Koscielniak [18]. However, under the customary assumption of a bell-shaped steady-state distribution, about which the expansion is made, the same wave theory predicts that self-sustaining perturbations are possible only (below transition) if the impedance is inductive or if the bell shape is inverted. 
In the following sections we describe measurements at the PSB and computer simulations of boosterlike beams, derive a Hamiltonian condition for stationarity, critique the linearized Vlasov equation, and find (almost) exact solutions of the nonlinear Vlasov-Poisson equation and self-consistency conditions. The stationarity conditions have scaling laws similar to the Keil-Schnell criteria, and all reduce to essentially the same condition of a Hamiltonian with bound states in a potential well.

\section{CERN PSB MEASUREMENTS}

The PSB is a proton synchrotron cycling between $50 \mathrm{MeV}$ and $1.4 \mathrm{GeV}$ kinetic energy. The ring, of radius $25 \mathrm{~m}$, has transition gamma $\gamma_{t}=4.15$ and the slip factor $\eta=\left(1 / \gamma_{t}^{2}-1 / \gamma_{s}^{2}\right)$ is equal to 0.843 at $50 \mathrm{MeV}$. The machine operates with a harmonic $h=1$ fundamental $\mathrm{rf}$ system and, in addition, there is an $h=2 \mathrm{rf}$ system and a high harmonic cavity capable of generating harmonics from $h=12$ to $h=26$ at the injection energy. Beam is injected over a few turns (from 0.1 to 12) from a $200 \mathrm{MHz}$ linear accelerator (linac). The beam energy spread varies with injected intensity from less than 400 to over $600 \mathrm{keV}$. The booster may also be operated in storage ring mode at $50 \mathrm{MeV}$; the rf systems may be either turned off, mechanically shorted at the gap, or operated with direct $\mathrm{rf}$ feedback to reduce the cavity impedance apparent to the beam. Despite these measures there is enough spurious or residual impedance to cause a mild coasting beam instability at intensities beyond $6 \times 10^{12}$ protons per pulse.

A wide-band beam pickup and a fast digital oscilloscope with deep memory are available for recording bunch shapes turn by turn, or once each $n$ turns. This data may be graphed as a waterfall display which may be considered a "bird's-eye" view of the more conventional mountainrange display. A gray scale is used to give a visual depiction of the relative particle density; black corresponds to the most dense and white to the most rarified. This instrument and software are called the "tomoscope." The low-level rf signal is available for frame synchronization purposes even when the cavities are turned off.

\section{A. Linac bubbles}

The tomoscope may be used to observe the debunched beam after injection. Figure 1 shows a typical display. Beam structure, which we refer to as "bubbles," survives for at least $50 \mathrm{~ms}$. This longevity could be ascribed to their having a very small momentum width, but this cannot be the complete explanation. Based on phase slippage alone, no appreciable debunching of the bubbles in $50 \mathrm{~ms}$ would imply a kinetic energy spread less than $5 \mathrm{eV}$, but this momentum width is so small that the bubbles would have no detectable effect on the line density. The bubbles become more prominent at higher intensity; see Fig. 2.

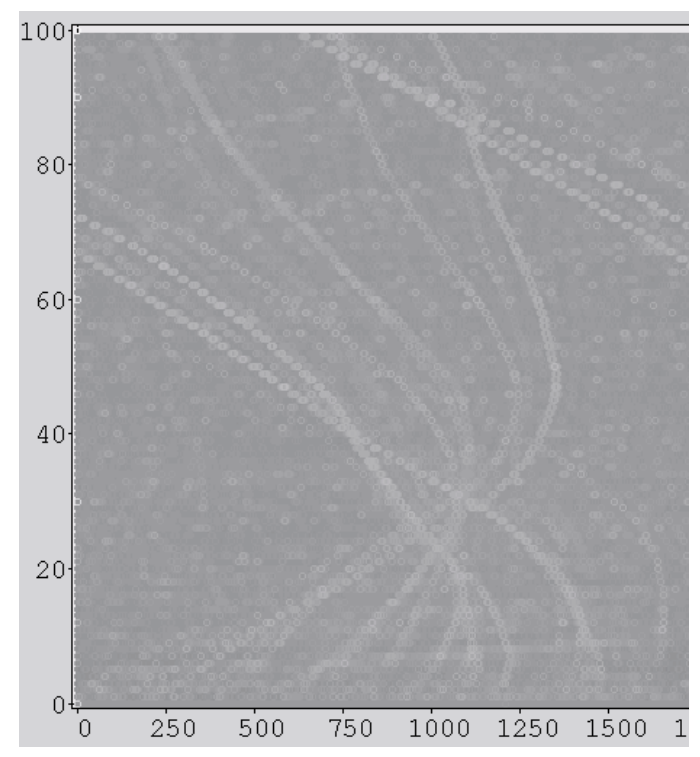

FIG. 1. $2.4 \times 10^{12}$ protons, one trace each 300 turns, vertical span $50 \mathrm{~ms}$; abscissa in nanoseconds (ns).

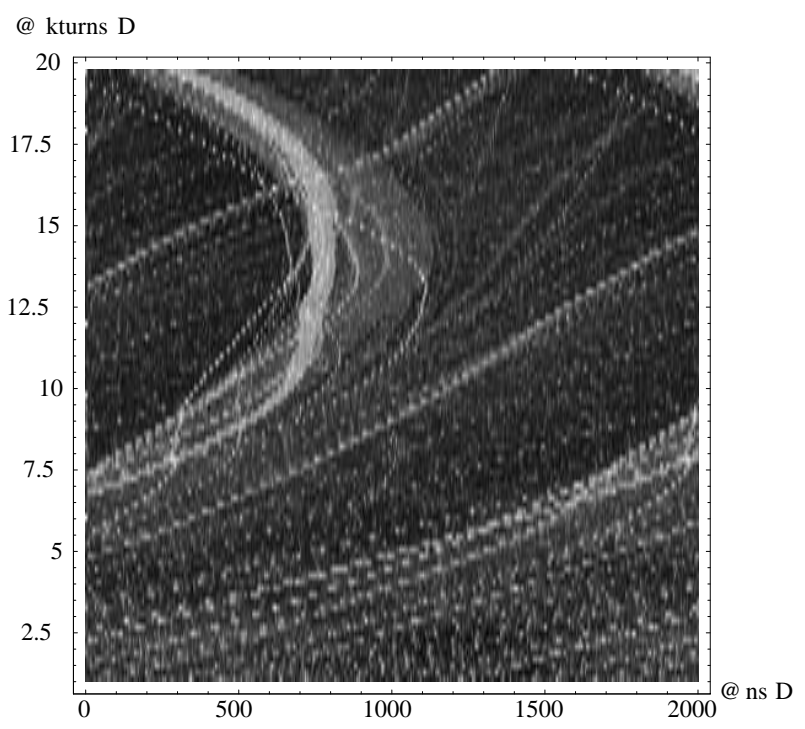

FIG. 2. $3.6 \times 10^{12}$ protons, one trace each 200 turns, vertical span $31.7 \mathrm{~ms}$.

\section{B. Refinement of observations}

The on-line tomoscope software uses small circles as a graphical primitive in forming the gray scale; this is a dangerous artifact when trying to observe bubbles. In order to give greater control over the visualization, the files were imported into MATHEMATICA [19] and graphed with a true gray scale. Figure 3 shows an example waterfall display in MATHEMATICA.

The diagonal movement of the tracks arises because the revolution frequency of the bubbles is not equal to the reference rf. This results in track wrap-around on the display 


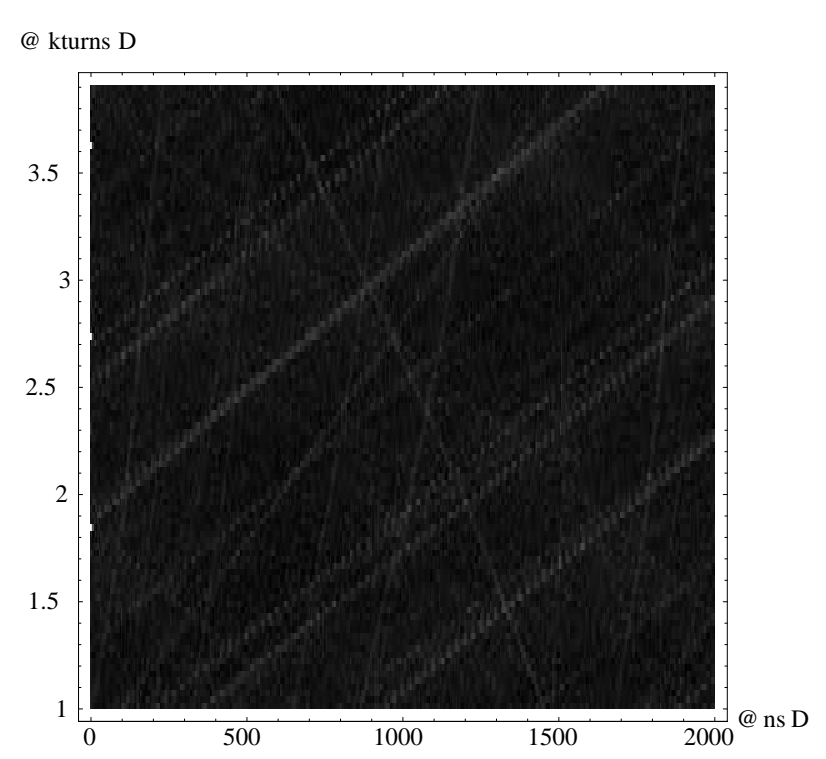

FIG. 3. $2.3 \times 10^{12}$ protons, one trace each 30 turns, vertical span $5 \mathrm{~ms}$.

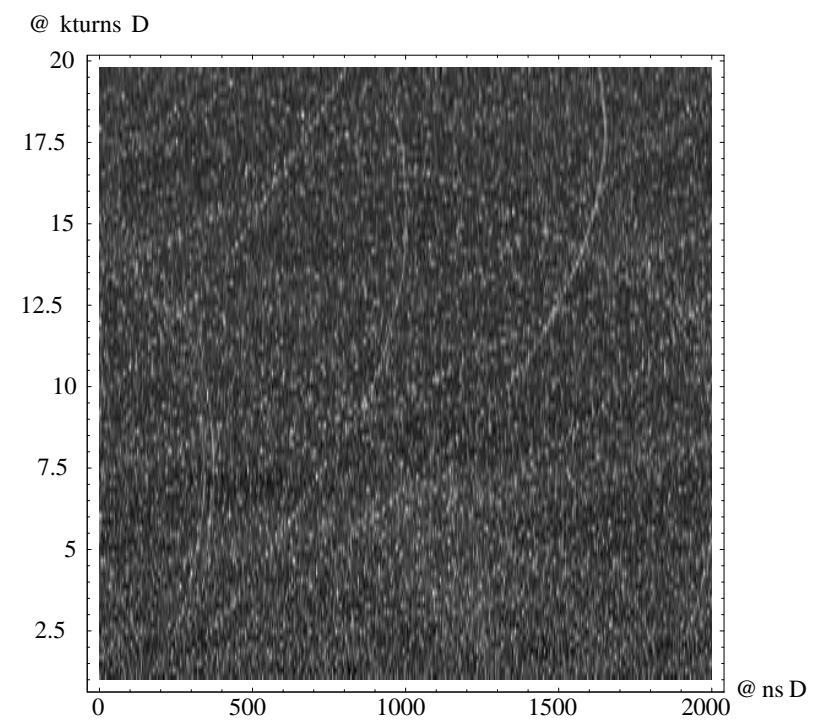

FIG. 4. $1.0 \times 10^{12}$ protons, one trace each 200 turns, vertical span $31.7 \mathrm{~ms}$.

and can be confusing. Fine adjustment of the rf may slow down the sideways drift, and this enables the time span to be increased. Figure 4 shows an example display. It is suspected that the bubbles emanate from perturbations in the linac beam current. Zooms of the data have confirmed that some holes are $10 \mathrm{~ns}$ long, consistent with $200 \mathrm{MHz}$ rf, but many are broadened.

\section{Schottky scans}

Further evidence for coasting beam bubbles comes from Schottky scans. Though one anticipates a noisy but basically smooth bell-shaped momentum distribution, what is seen is numerous spikes superposed on a quasiparabolic background; see Fig. 5. Whereas the true incoherent Schottky signal is proportional to the square root of particle density per unit frequency interval, coherent signals due to bubbles are directly proportional to the number of particles involved; and so coherent spikes can dominate the spectrum. Figure 6 shows a waterfall display taken shortly after the Schottky scan.

The linac bubbles arrive without prescribed properties; thus one can do little but observe their longevity and their propensity to cross but not coalesce. In order to compare quantitatively against some theoretical model, one needs holes with predictable and controllable properties. Fortunately, in the PSB we have at our disposal a high harmonic cavity with which to introduce holes of our own making.

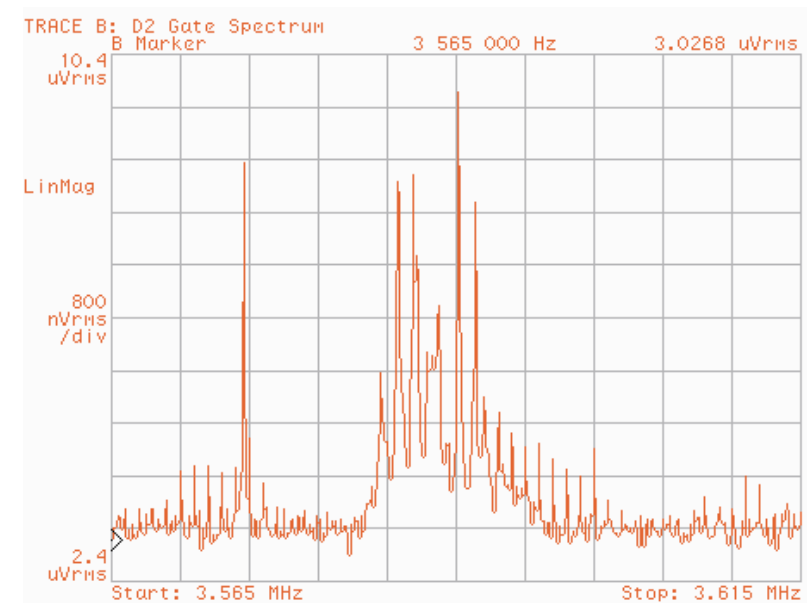

FIG. 5. $1.7 \times 10^{12}$ protons, Schottky scan about $h=4$.

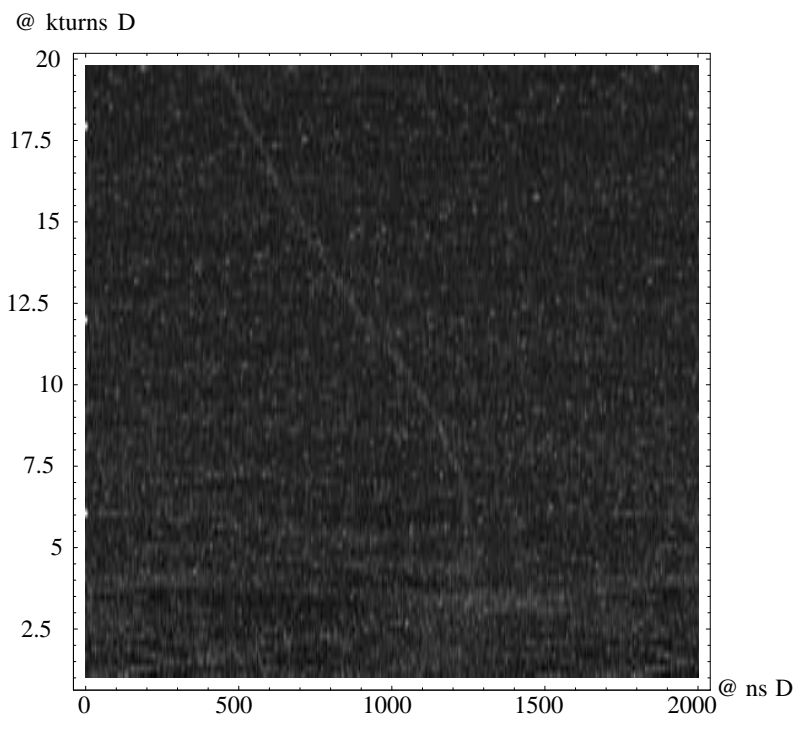

FIG. 6 . $1.7 \times 10^{12}$ protons, one trace each 200 turns, vertical span $31.7 \mathrm{~ms}$. 


\section{D. rf capture}

The impetus to study holes in debunched beams at the PSB arose from a desire to create bunches which are hollow in longitudinal phase space and whose projection over momentum gives flat bunch shapes. Using a high harmonic cavity with swept frequency, the technique [20,21] is to deposit empty rf buckets into the core of a debunched beam thereby creating a double-peaked momentum distribution, capture this beam into a single bucket at the rf fundamental, and then accelerate. There were two surprises: (i) the phase-space voids introduced by empty buckets do not debunch and (ii) there are pulse-to-pulse variations in the $h=1$ bunch at the time of capture. The second effect has since been diagnosed as piratical disruption of the nascent $h=1$ bunches by linac bubbles. The first effect is the subject of this paper. Figure 7, left side, shows a debunched beam with linac bubbles captured into an $h=1 \mathrm{rf}$ bucket. Figure 7, right side, shows the introduction of high harmonic empty buckets, which gives a periodic density modulation, the pirate linac bubbles, and the rf capture.

\section{E. Periodic high-harmonic holes}

If the fundamental rf is turned off and the ring is operated in storage mode, the same high harmonic cavity can be used to introduce periodic holes with known properties. Actually, space charge will distort the empty rf buckets making for some uncertainty, but this is a not too significant effect. Figure 8 shows periodic holes introduced at

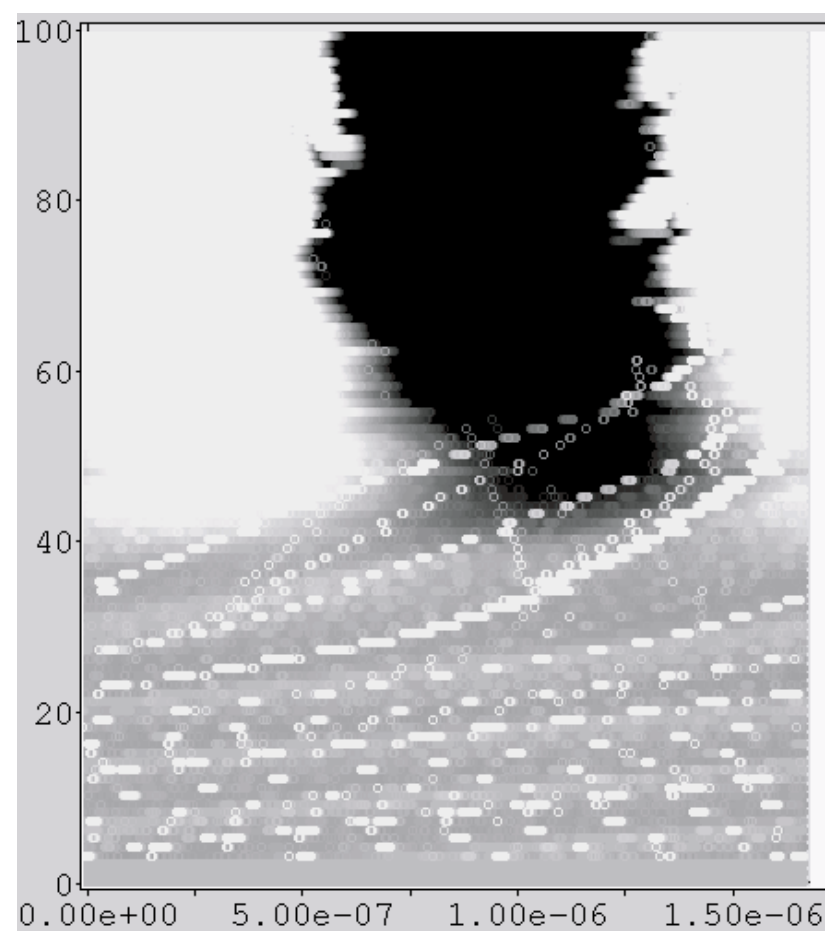

$h=13$ with a cavity voltage of $3 \mathrm{kV}$. The quasiparabolic sweep of the tracks is due to variation in the rf program which was set somewhat crudely.

In the absence of focusing forces, the $h=13$ holes should shear in longitudinal phase space; their kinetic energy spread (computed from rf bucket parameters) is some $190 \mathrm{keV}$. During an interval of $8.3 \mathrm{~ms}$, the top and bottom of the $h=13$ empty rf buckets should shear by some 8.3 periods of rf phase at $h=1$, but no such shearing can be detected.

Space charge is the dominant longitudinal impedance in the PSB and has been identified as the likely explanation for the preceding phenomena.

\section{F. Momentum steering of holes}

Because the longitudinal space-charge force [15] is proportional to the derivative of the line-current density, there is no force of attraction or repulsion between holes until they contact. However, if there are small differences in their central momenta, then holes will move relative to one another. If the differences are random, some holes will move closer together while others will move farther apart. Typically this is a slow process because the momentum differences are small. But eventually two or more holes will collide.

As a test of this idea, one can hope to impress a long wavelength coherent energy modulation on the hole centers so that the collision process is speeded up and not governed

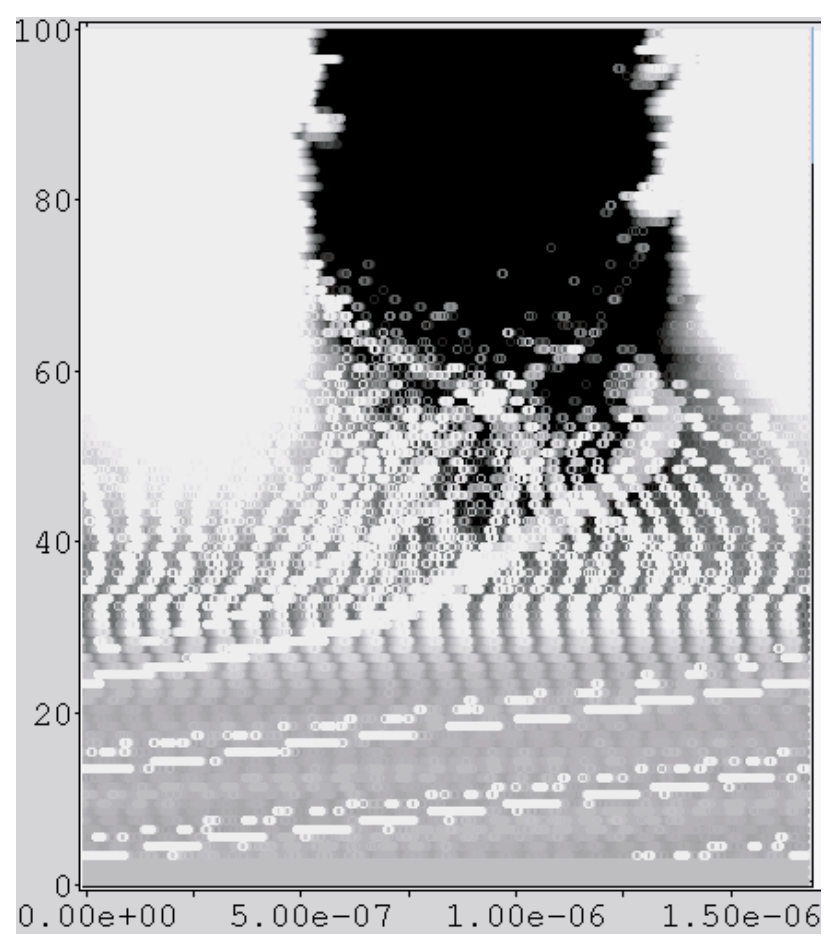

FIG. 7. Waterfall plots of rf capture with (right) and without (left) high-harmonic bucket deposition. $3.2 \times 10^{12}$ protons, one trace each 20 turns, vertical span $3.3 \mathrm{~ms}$. 

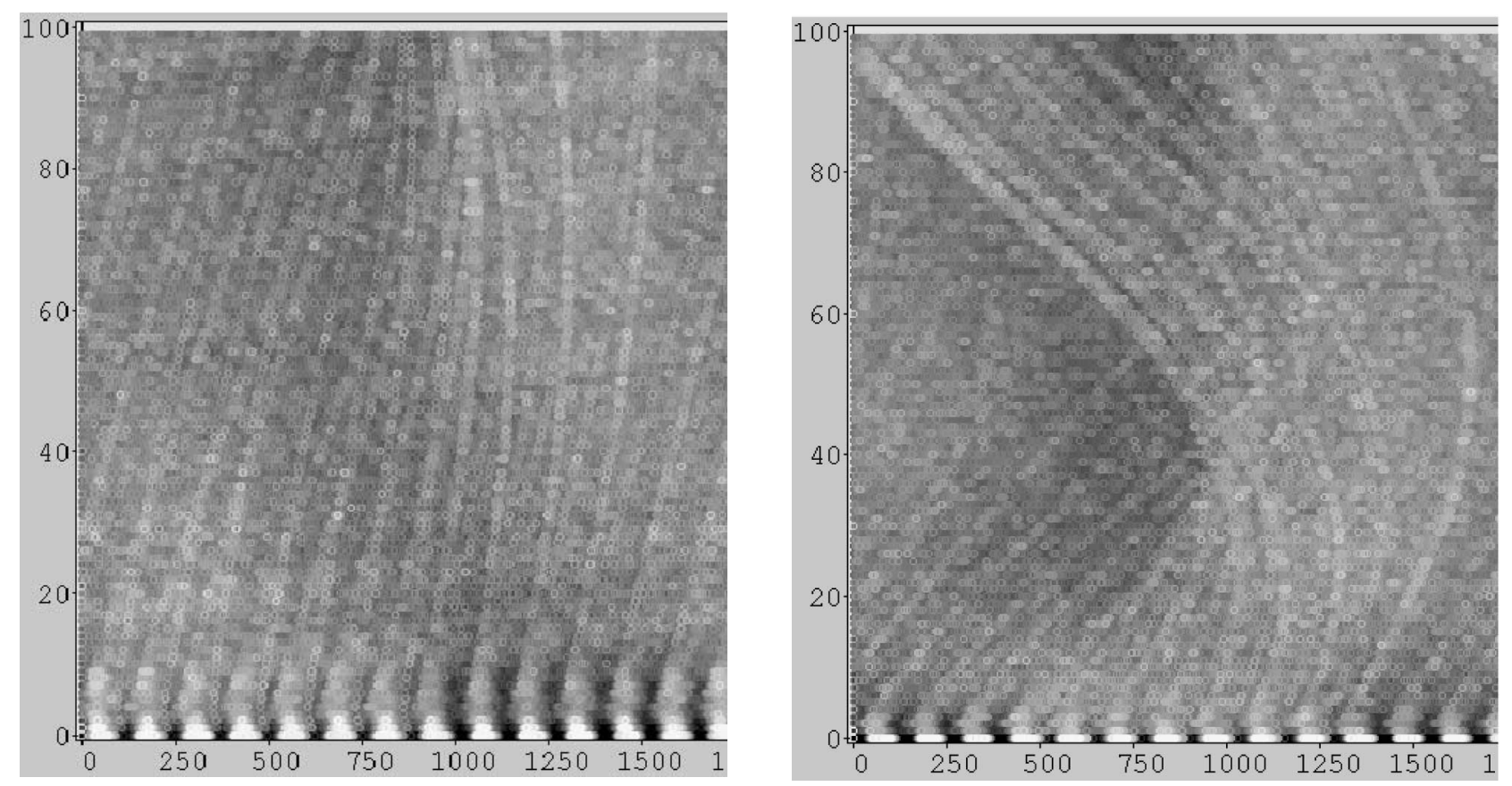

FIG. 8. Waterfall plots of line density. Left: one trace each 20 turns, span $3.3 \mathrm{~ms}$. Right: one trace each 50 turns, span $8.3 \mathrm{~ms}$; abscissa in nanoseconds. $3.0 \times 10^{12}$ protons.

by randomness. Hence one is led to modulating the holes at the revolution frequency with a small voltage of short duration. Figure 9 shows typical waterfall displays. The tracks of the holes converge in response to the modulation and though some holes collide none coalesce.

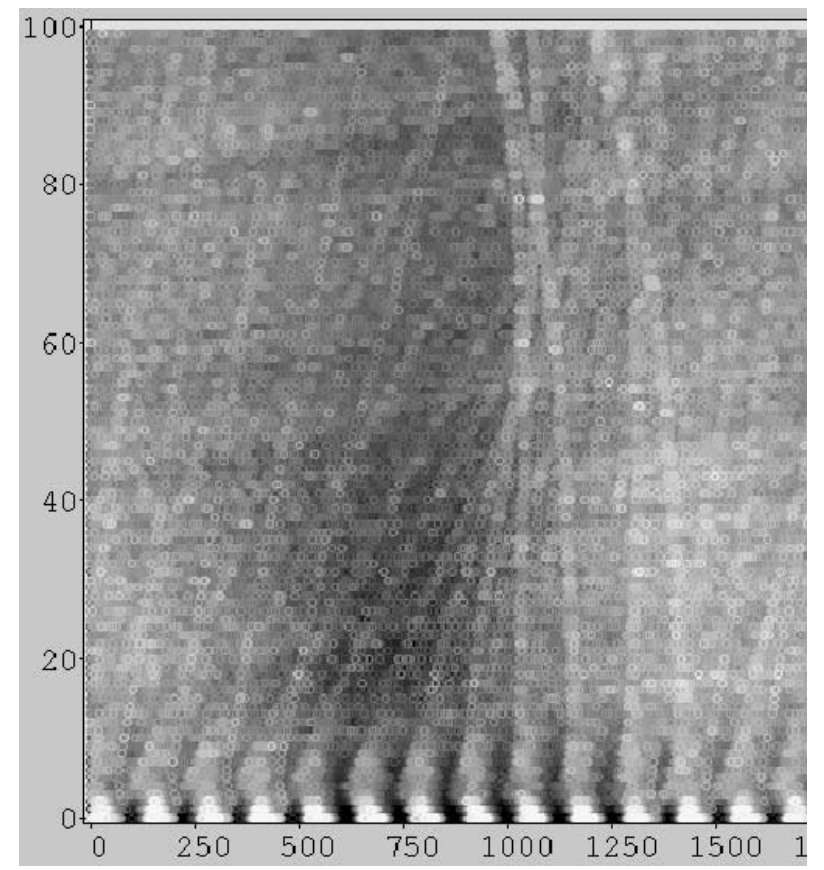

\section{G. Momentum spread versus beam current}

A simple theoretical model (see Sec. III) was developed in parallel with the later beam measurements at the booster, and these experiments were adapted to test the

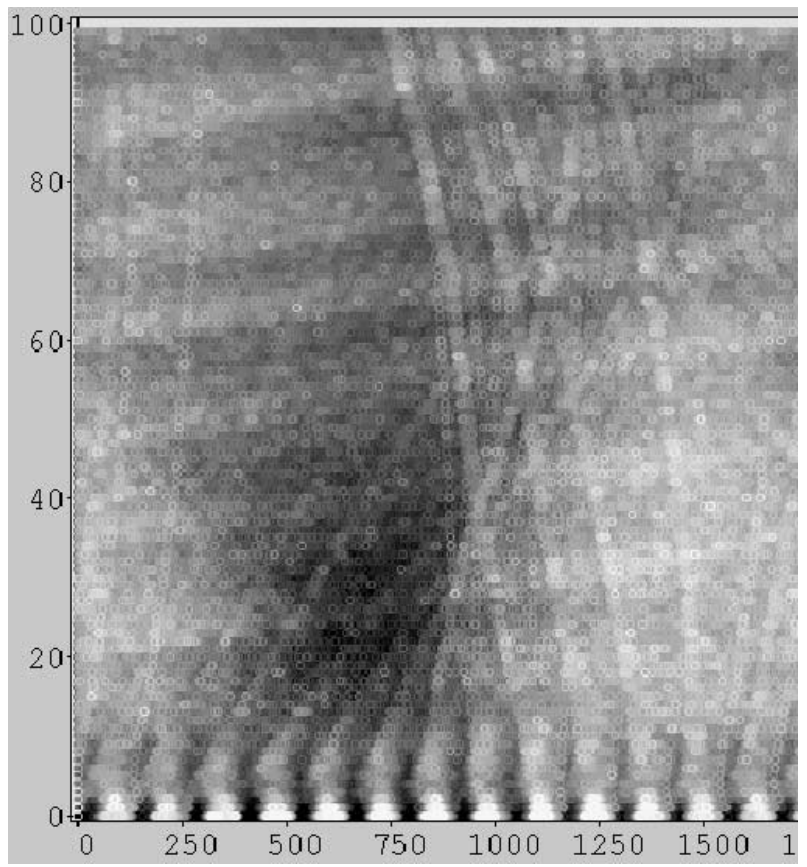

FIG. 9. Periodic holes with $\pm 60 \mathrm{keV}$ (left) and $\pm 120 \mathrm{keV}$ (right) energy modulation at $h=1$. One trace each 20 turns, span $3.3 \mathrm{~ms}$; abscissa in nanoseconds. $2.4 \times 10^{12}$ protons. 
@ kturns D

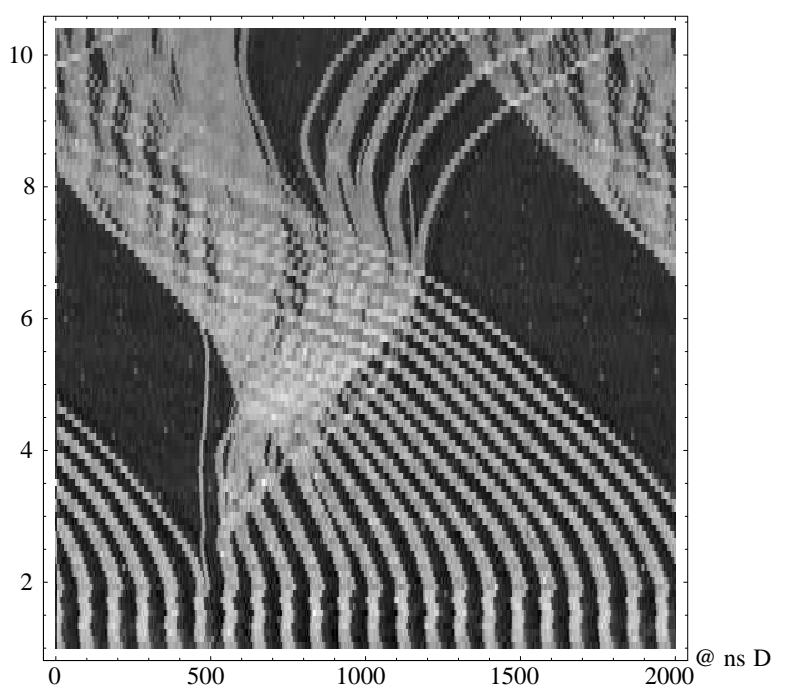

FIG. 10. $1.8 \times 10^{12}$ protons, one trace each 100 turns, vertical span $16.7 \mathrm{~ms}, V_{\mathrm{rf}}=0.3 \mathrm{keV}$.

theory which predicts a definite relation between the momentum spread of the holes and the beam current because of a fine balance between shearing and focusing. In these measurements, the harmonic cavity was run at $h=18$.

In Fig. 10, the tendency for holes to shear due to their momentum spread is well balanced against the focusing provided by space-charge forces, and the tracks stay sharply defined. It is also clear that although some holes appear to collide they pass one another and do not coalesce. In Fig. 11, the momentum spread of the holes has been enlarged (cavity voltage doubled) and this enhances the shearing effect.

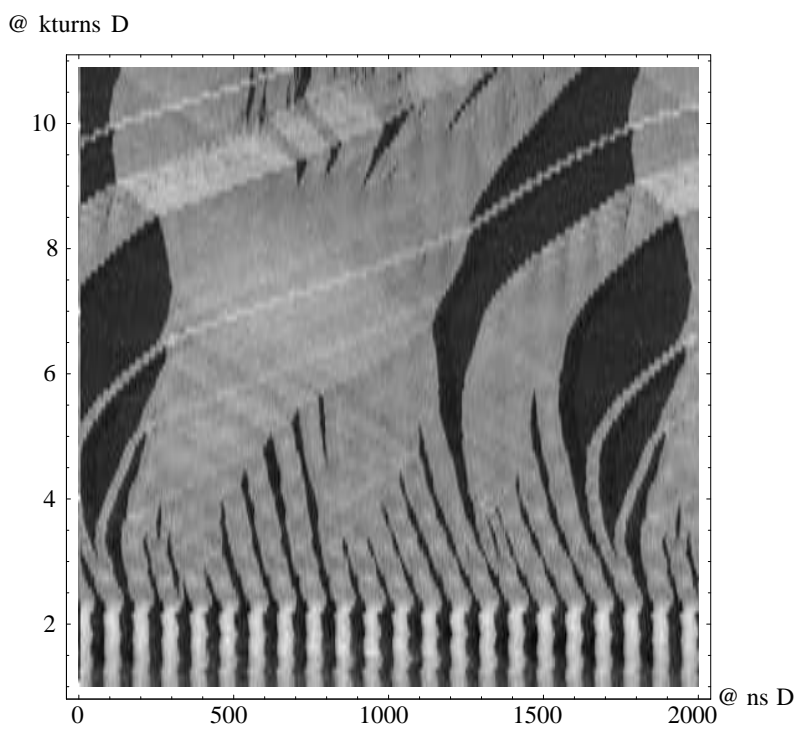

FIG. 11. $1.8 \times 10^{12}$ protons, one trace each 100 turns, vertical span $16.7 \mathrm{~ms}, V_{\mathrm{rf}}=0.6 \mathrm{kV}$.
@ kturns D

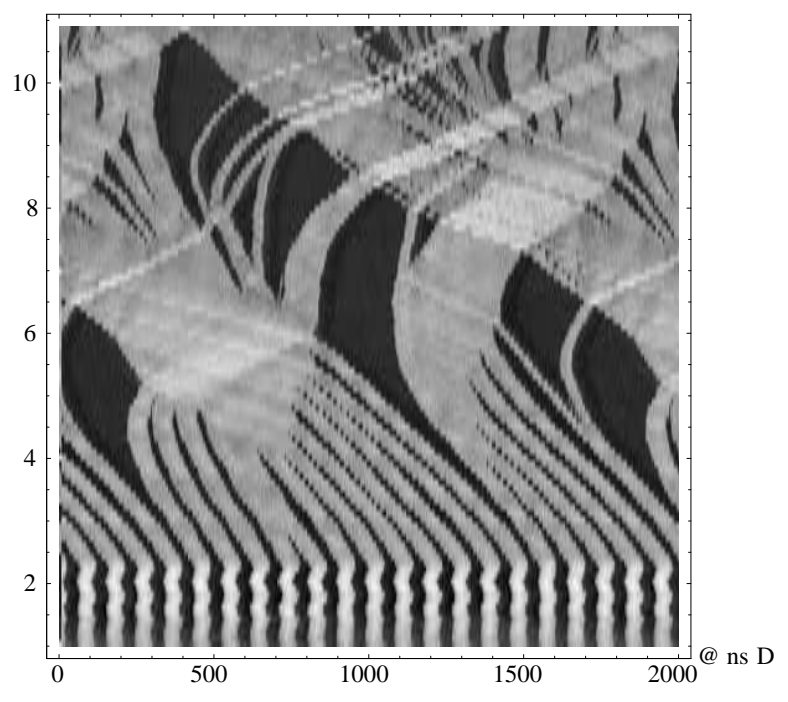

FIG. 12. $2.4 \times 10^{12}$ protons, one trace each 100 turns, vertical span $16.7 \mathrm{~ms}, V_{\mathrm{rf}}=0.55 \mathrm{kV}$.

A comparison of Figs. 11-13, where the beam current is progressively increased, shows that space-charge focusing on holes can overcome the shearing due to momentum spread, leading to narrower and more self-sustained holes. The affect of phase-space displacement acceleration also depends on intensity, because of bucket distortion, and so the final central energy of the holes varies. And, since the rf acquisition frequency was not adjusted to compensate, this effect is responsible for the difference in the phase-slip rates between the figures.

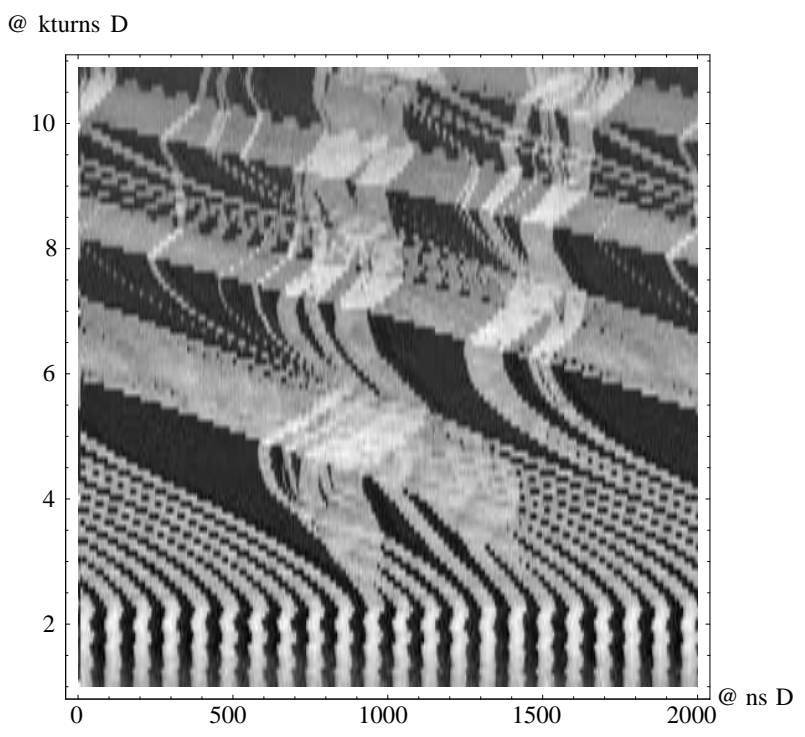

FIG. 13. $3.2 \times 10^{12}$ protons, one trace each 100 turns, vertical span $16.7 \mathrm{~ms}, V_{\mathrm{rf}}=0.6 \mathrm{kV}$. 

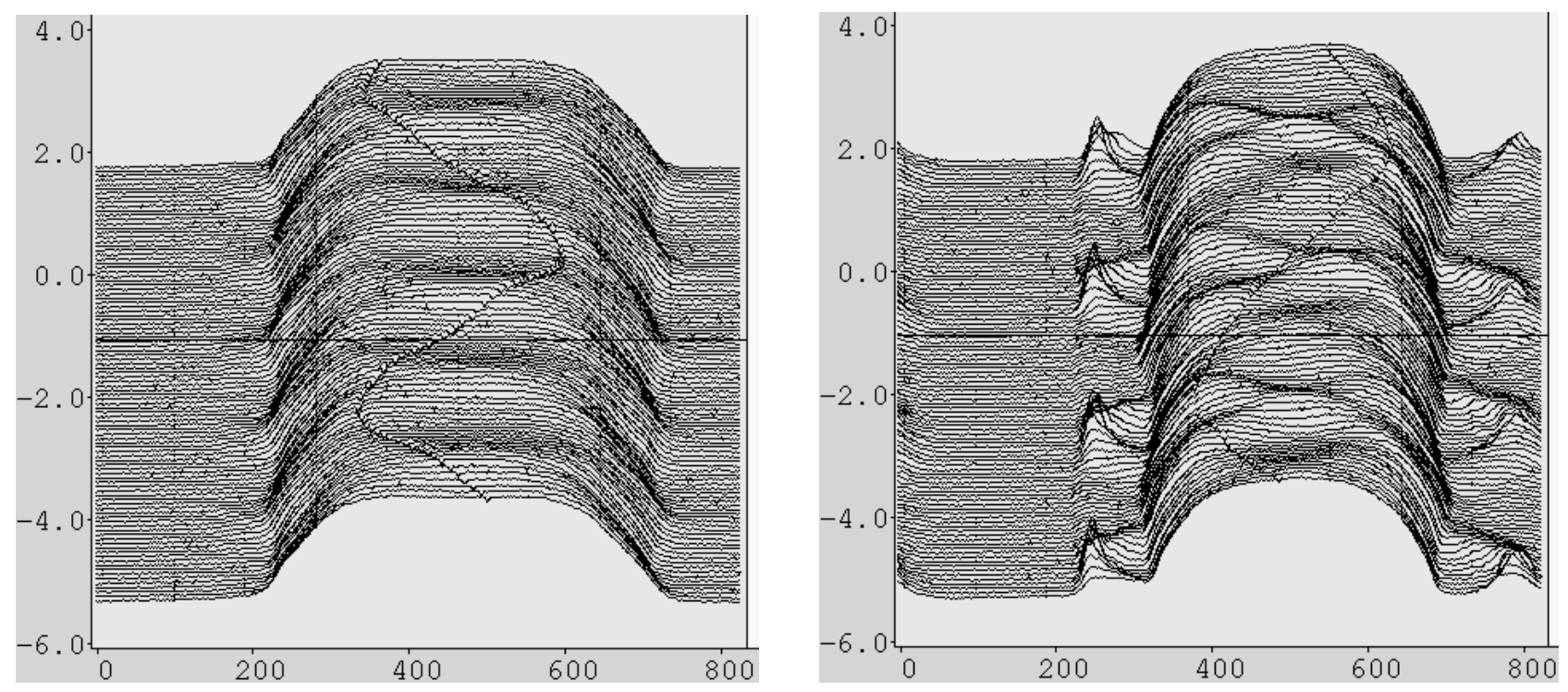

FIG. 14. Mountain-range plots of line density. Left: start at $162 \mathrm{~ms}$, one trace each 25 turns, span 4.0 ms. Right: start at $202 \mathrm{ms,}$ one trace each 20 turns, span $3.1 \mathrm{~ms}$. Abscissa in nanoseconds. $5.5 \times 10^{12}$ protons. Dual harmonic with $V_{2} / V_{1}=0.5$.

\section{H. Holes in bunched beams}

There is some evidence that small phase-space voids survive capture and acceleration in the PSB. Typically there is a spread in synchrotron oscillation frequencies which will cause a beam perturbation to filament. Consequently, one expects to find that holes must be small and that they survive where the frequency spread is smallest. Single holes at the very center of a single harmonic rf bucket have been found [22] to survive right through to extraction to the CERN PS. For the case of dual-harmonic acceleration, the synchrotron frequency $\omega_{s}$ first increases and later decreases with amplitude $r$, and there is a particular amplitude at which the derivative $d \omega_{s} / d r=0$. Holes performing incoherent oscillations of this particular amplitude have been found to survive for at least $200 \mathrm{~ms}$; see Fig. 14. Such motions can be used as a clock against which the period of coherent bunch oscillations may be timed.

\section{SIMPLE THEORETICAL MODEL}

In support of the CERN PSB experiments and the computer simulations at TRIUMF, a simple criterion for the stationarity and self-consistency of a hole was developed by consideration, in detail, of the phase-space trajectories about a rectangular void. Let us suppose that "space charge" is the dominant longitudinal impedance, as is usually the case in low energy proton synchrotrons. For simplicity, we shall suppose that the central momentum of the void is equal to that of the beam, so that the perturbation moves neither to the right nor to the left. Let $p$ and $q$ be canonical momentum and azimuthal position coordinates; their relation to world coordinates such as rf phase $\phi$ and energy deviation $\Delta E$ are given in the Appendix. The
Hamiltonian is $H=p^{2} / 2+Z \lambda(q)$ where $Z>0$ is a coupling constant and $\lambda$ is the line density.

\section{A. Physical picture}

For the purpose of clarity we shall discuss a phase-space hole that resembles a small, empty barrier bucket embedded in a much larger background of charges. Consider Fig. 15. We take the line density constant over the region $a_{1} \leq q \leq a_{2}$ and falling and rising, respectively, over the transition regions $b_{1}<q<a_{1}$ and $a_{2}<q<b_{2}$ where $b_{1}<a_{1}<a_{2}<b_{2}$. The line density is constant elsewhere. The variation in $\lambda$ gives rise to a potential function with the same shape as $\lambda$. In this case, below transition energy, we have a potential well which captures the empty phase space. The precise variation of $\lambda$ over the transition regions is unimportant, since the net change in $p^{2}$ during crossing depends only on the potential differences $\Delta \lambda_{1,2}=\lambda\left(b_{1,2}\right)-\lambda\left(a_{1,2}\right)$. Particles receive equal and opposite space-charge impulses as they cross the two transition regions, and it is these impulses which may maintain the shape of the hole. The sequence of two impulses is such that the modulus of the momentum excursion first increases and then decreases. Particles which have initially small momenta have their orbits distorted substantially by these impulses and trace out paths that flow around the hole and delineate its boundary. Particles with initially large momenta $\left(p_{0}\right)$ receive the same impulses but have their paths much less distorted because $p=\sqrt{p_{0}^{2}+Z \Delta \lambda(\phi)}$. Hence, if the beam momentum spread is sufficiently large, the boundary of initially occupied phase space is hardly distorted by the introduction of a hole. Particles which are on the separatrix of the hole (and nonparticles inside the hole) receive double impulses in each of the transition 

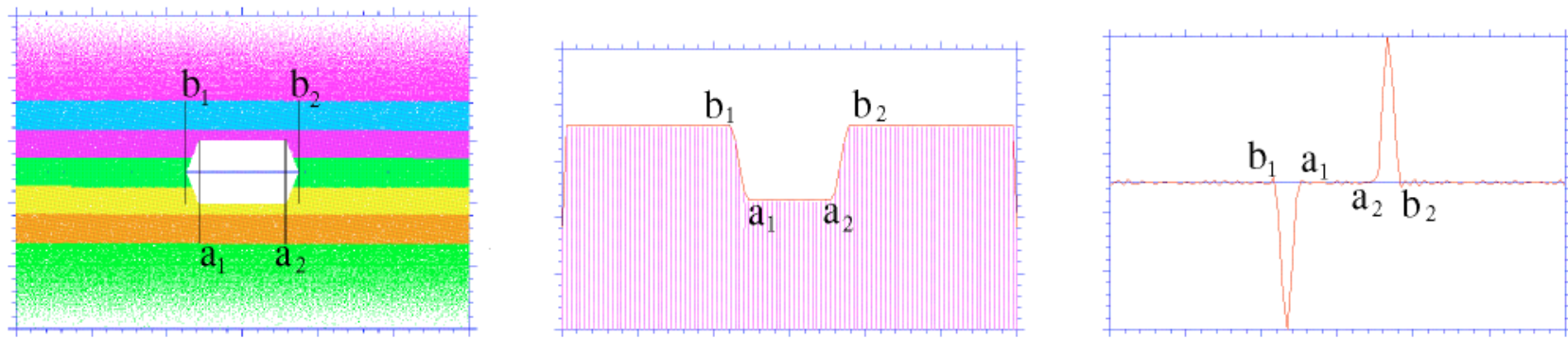

FIG. 15. (Color) Phase space, line density, and space-charge voltage for single hole.

regions (and have their directions of motion reversed) and so circulate about the hole center rather than flowing around the hole. This discontinuous behavior is characteristic of a separatrix.

\section{B. Equilibrium condition}

Let us suppose the empty bucket is cut away from an overall background momentum distribution $F(p)$ which is independent of $q$ and extends from $-\Delta p$ to $+\Delta p$. The momentum-deviation shape of the hole on upper and lower contours is denoted $\pm \hat{p}(q)$; the implied symmetry occurs because all forces acting are conservative. Hence, the line density is $\lambda(q)=\lambda_{0}+\lambda_{1}(q)$, where

$$
\begin{aligned}
\lambda_{0} & =+\rho_{0} \int_{-\Delta p}^{+\Delta p} F(p) d p \\
\text { and } \quad \lambda_{1}(q) & =-\rho_{0} \int_{-\hat{p}(q)}^{+\hat{p}(q)} F(p) d p .
\end{aligned}
$$

The constant $\rho_{0}$ is determined by the normalization condition $\int_{-\pi}^{+\pi} \lambda(q) d q=N$, where $N$ is the number of particles.

At the ends of the empty barrier bucket, $p=0$ and $\lambda_{1}(|b|)=0$ and so $H=0$. Hence, the momentum variation along the bounding contour of the hole is

$$
p^{2} / 2=-Z \lambda_{1}(q)=2 Z \rho_{0} \int_{0}^{\hat{p}(q)} F(p) d p .
$$

But since this contour is the perimeter of the hole, selfconsistency demands that $p(q)=\hat{p}(q)$. Hence we have an equation of the form $p^{2}(q)=G[p(q)]$, which has two possible solutions: (i) $G=p^{2}$ and $F(p) \propto p$, which is unphysical, or (ii) $a \rightarrow b$ and

$$
(\hat{p})^{2}=4 Z \rho_{0} \int_{0}^{\hat{p}} F(p) d p .
$$

Hence we conclude the self-consistent solution of the product form $\psi_{1}=P(p) Q(q)$ is a rectangular hole with space-charge forces confined to positive and negative Dirac impulse functions at either end and, moreover, that the momentum spread of the hole be consistent with the beam current according to Eq. (3), which expresses a competition between a tendency for the hole to shear because of its momentum spread versus space charge which attempts to focus the motion toward the hole's center. For holes which are close to the rectangular shape, one still expects the maximal momentum to satisfy condition (3).

\section{Scaling laws}

Equation (3) may be rewritten to explicitly show the scaling laws for fundamental parameters. We consider a particle beam of energy $E_{s}$, synchronous momentum $P_{s}$, and speed $\beta_{s} c$ moving in a storage ring of average radius $R_{s}$ and a hole of momentum width $2 \hat{p}$ and length $2 b$ rad. Let $g_{0}$ be the geometrical coupling parameter which depends on the ratio of beam and vacuum pipe transverse radii. The equilibrium half-height momentum spread of the hole is

$$
\begin{aligned}
\left(\frac{\hat{p}}{P_{s}}\right)^{2}= & \frac{g_{0}}{2 \pi \epsilon_{0}}\left(\frac{e}{\beta_{s}}\right)^{2} \frac{1}{E_{s}} \frac{N}{R_{s}} \frac{1}{\left[\left(\gamma_{s} / \gamma_{t}\right)^{2}-1\right]} \\
& \times \frac{f_{\text {empty }}}{f_{\text {filled }}} \frac{1}{2 b},
\end{aligned}
$$

where $f_{\text {empty }}$ and $f_{\text {filled }}$ are, respectively, the fractions of empty and filled phase space.

$$
\frac{f_{\text {empty }}}{f_{\text {filled }}}=\frac{b \int_{0}^{\hat{p}} F(p) d p}{\pi \int_{0}^{\Delta p} F(p) d p-(b / h) \int_{0}^{\hat{p}} F(p) d p} .
$$

The scaling law is independent of harmonic number, as may be anticipated for an unbunched beam. The dependence on $\beta_{s}$ and $E_{s}$ implies the effect will be more prominent at low energy. The rather complicated form for $f_{\text {empty }} / f_{\text {filled }}$ derives from the fact that we insisted not to change the total number of particles $N$ when the hole was "cut away" from the background of charges.

\section{Simplifying approximations}

We introduce two approximations into Eqs. (1)-(3) to simplify the expressions.

(i) Sufficiently small hole that $2 \pi \rho_{0} \int_{-\Delta p}^{+\Delta p} F(p) d p \rightarrow$ $N$.

(ii) $F(p)$ slowly varying and $\hat{p} \ll \Delta p$.

The half-height momentum spread of the hole is approximately

$$
\hat{p} \approx \frac{2 Z}{\pi} F(0) / \int_{-\Delta p}^{+\Delta p} F(p) d p .
$$

This stationarity condition in world coordinates and SI units is 


$$
\begin{aligned}
\left(\frac{\hat{p}}{P_{s}}\right) \approx & \frac{g_{0}}{2 \pi \epsilon_{0}}(e)^{2} \frac{1}{\beta_{s} c} \frac{N}{R_{s}} \frac{1}{\left[\left(\gamma_{s} / \gamma_{t}\right)^{2}-1\right]} \\
& \times \frac{F(0)}{2 \pi \int_{0}^{\Delta p} F(p) d p} .
\end{aligned}
$$

The computer simulations of single holes (see Sec. IV B 1) confirm this matching criterion. The PSB experiments with periodic holes introduced by a high-harmonic rf (Sec. II G) are also in agreement with this formula, though the depth of the holes is not a precisely known quantity.

\section{E. Away from equilibrium}

From the above scaling law, one may speculate on the behavior of holes away from equilibrium. If the momentum spread is too large, the hole shears along the $q$ direction, the width of the transition region increases, the space charge forces diminish still further, and the shear rate increases, and so the hole filaments and disappears. If the momentum spread is too small, the two transition regions shear along the $p$ direction in such a way as to increase their momentum spread. This in turn will create two cusps in the line density, which generate positive and negative space-charge impulses at either end of both transition regions. This in turn redistributes particles so that the bunch splits in two.

\section{F. Collision of two holes}

Two holes can only move relative to one another if their central momenta are different. Further, they can only collide in phase space if their momentum difference is less than the sum of their half-width momentum spreads. We shall consider two holes which are matched according to condition (3) and are identical except for their initial locations and central momenta: a lower momentum hole on the left (traveling rightward) and a higher momentum hole on the right (traveling leftward); see Fig. 16. We take a reference frame in phase space in which the holes move with equal and opposite speeds toward one another. One possible "picture" for the moving phase space trajectories at the boundary of each hole is that of two caterpillar tracks, with the key observation that "although different parts of a track move in different directions, nevertheless the track as a whole moves forward (or backward)." The higher momentum hole has a right leading edge (A) and a left trailing edge (B), whereas the lower momentum hole has a left leading edge (C) and a right trailing edge (D). Associated with these four edges are transition regions where $\lambda$ varies and particles receive space-charge impulses. There are positive impulses at edges $\mathrm{B}$ and $\mathrm{C}$ and negative impulses at edges $\mathrm{A}$ and $\mathrm{D}$, and these impart a counterclockwise sense of rotation to the nonparticles inside the holes.

The edges $\mathrm{A}$ and $\mathrm{C}$ are the first to encounter one another. Whether the edges $\mathrm{A}$ and $\mathrm{C}$ collide in phase space or merely pass by one another, the result is much the same. When and where (in phase) the A and $\mathrm{C}$ transition regions collide, the $\lambda$ variations cancel exactly, the spacecharge forces drop to zero, and (locally) particles are free to drift - which leads to a slight shearing of the edges A and $\mathrm{C}$.

After the $\mathrm{A}$ and $\mathrm{C}$ transition regions have passed through one another, the space-charge forces in the A transition region will continue to act on the lower momentum hole and, likewise, forces at the $\mathrm{C}$ edge will act on the higher momentum hole and particles that surround it. The impulses, which are half the values continuing to be applied at the edges $\mathrm{B}$ and $\mathrm{D}$, are repulsive and so the effect of these shock waves is to distort the holes so that they avoid intimate encounter. At the same time, occupied phase space is introduced between the two holes to form a "boundary layer."

The two holes continue to move relative to one another; eventually and inevitably the edges A and D will collide, and at the same moment the edges B and C collide. The $\lambda$ variations will superpose constructively so that the spacecharge forces at either end are (almost) doubled. If the initial momentum separation is small, this gives a situation which is very similar to that of a single hole of twice the height (and area) of our two initial holes. The space charge has doubled, and the momentum width has at least doubled compared with that of a single hole. This implies that the self-consistency condition (3) is not fulfilled, but rather the momentum spread is a factor $\sqrt{2}$ too great and that the "new hole" will shear. Alternatively, we may say that the two holes continue to slide past one another allowing the edges B and D to generate impulses which (almost) reverse all of the actions earlier performed by the edges A and D.
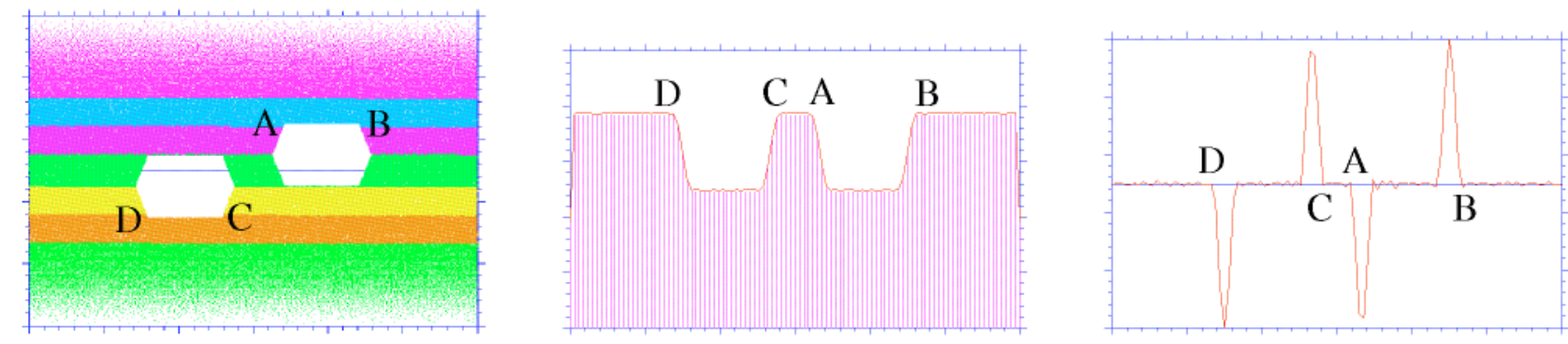

FIG. 16. (Color) Phase space, line density, and space-charge voltage for two holes. 
In total, this series of events implies that, even if the initial separation of hole centers is much less than their width, they still do not actually collide or coalesce, but rather flow around one another. This is comforting because true collisions of phase-space trajectories are forbidden in Liouvillian flows.

Note that these remarks on the collision of matched holes are similar to those of Laclare [17] who states, in the context of rf stacking, "the elementary stacks are potentially more unstable than the final accumulated beams."

\section{COMPUTER SIMULATIONS}

In support of the experiments at the PSB, many computer simulations were made using the LONG1D [23] macroparticle tracking program. The simulation has several benefits: conditions can be controlled precisely, more views of the beam (such as phase space density and momentum spectrum) are available, and the impedance can be precisely defined. LONG1D, which uses a secondorder symplectic multistep algorithm for handling of the space-charge dynamics, computes the space-charge [24] electric field based on Fourier analysis of the line density and includes the roll off at high frequency and dynamical effects.

Two types of system were studied: (i) a periodic array of holes as the result of depositing empty rf buckets and (ii) the behavior of one or two rectangular holes. Examples of gif animations for both types may be found on the World Wide Web [25].

\section{A. Periodic holes}

A periodic array of holes was generated by simulating the deposition of high harmonic empty buckets into a debunched beam. The basic scenario is as follows: the cavity is turned on with $2 \mathrm{kV}$ voltage and with a frequency offset of $40-45 \mathrm{kHz}$ above the revolution harmonic; after $0.5 \mathrm{~ms}$ the frequency offset is ramped down to zero during a further $0.5 \mathrm{~ms}$; after this time the cavity is turned off and the beam evolution is tracked for a further $9.0 \mathrm{~ms}$.

Studies were made using harmonic number $h=15$ and $h=20$. Although the results were similar, the $h=15$ case is reported here for several reasons. The resolution of the plots is better at lower harmonic number, the statistics are better because there are more macroparticles per wavelength, and because the Fourier expansion of the space-charge wake is truncated to 50 harmonics there is more "head room" when the beam structure has lower periodicity.

Tests were also made on computational aspects. Repeated simulations were made with progressively more (i) macroparticles from $10^{5}$ to $10^{6}$ and (ii) time steps per turn from 1 to 3. Apart from the reduced noise there was no qualitative change in the behavior of the simulated particle beam.

\section{Variation with intensity}

The zero intensity case (Fig. 17) shows the holes to debunch in less than $0.5 \mathrm{~ms}$, and thereafter the beam is completely uniform as indicated by the constant value of the gray scale. The slip factor $\eta=0.843$ in the booster at $50 \mathrm{MeV}$ is comparatively large and this explains the rapid debunching.

In the case of $2 \times 10^{12}$ protons (Fig. 18, left side), the holes leave obvious tracks though space charge is not strong enough to prevent the holes from widening. Diagonal movement of some of the holes indicates that their revolution frequency and central momentum differ from the nominal. Some of the tracks converge and others diverge because of relative momentum differences between the holes; these differences arise purely from statistics and the movement tended to be faster when fewer macroparticles were used. Figure 18, right side, is a waterfall display of the beam momentum spectrum during the empty bucket deposition and subsequently. The dip in the central density is due to the projection over all 15 holes.

In the case of $4 \times 10^{12}$ protons (Fig. 19, left side), all the holes have revolution frequency substantially different from the nominal value. Space-charge forces distort the rf bucket shapes during the deposit of empty phase space, and this implies the final energy of the holes depends on beam current. The higher space-charge forces, cf. the previous case, also provide better focusing of the holes and so they do not widen. Figure 19, right side, shows a spectrogram of the frequency content versus turns. The gray scale is proportional to the square root of the Fourier amplitude so as to reveal the weaker Fourier components. The $h=15$

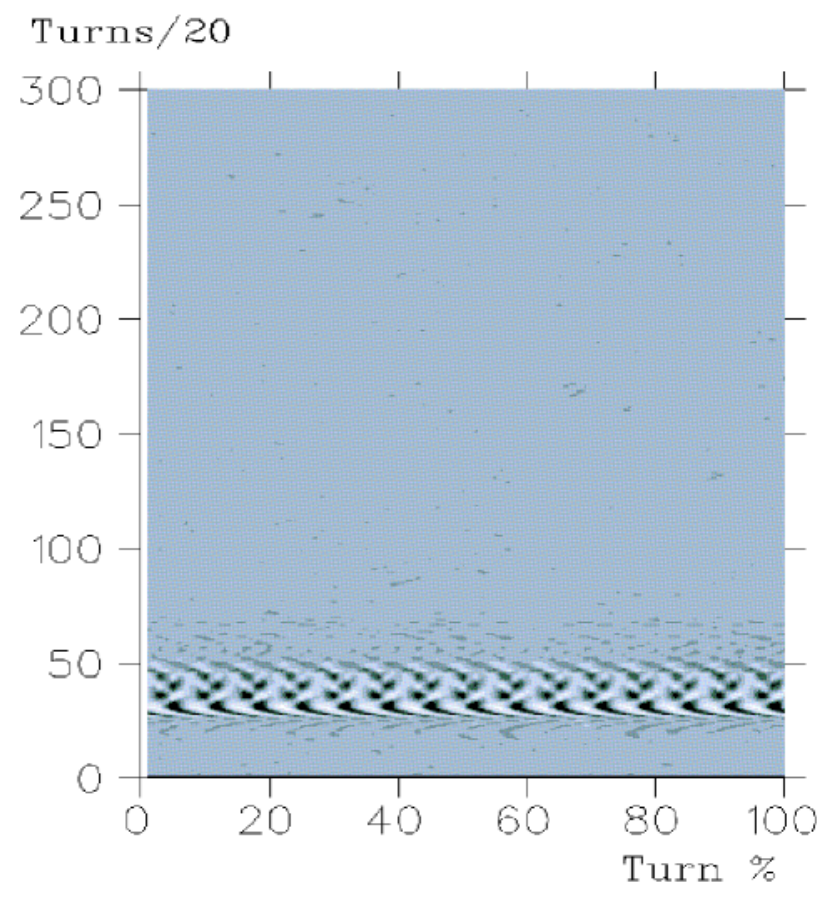

FIG. 17. Waterfall plot of line density, 6000 turns, 0 protons, $10^{6}$ macroparticles. 

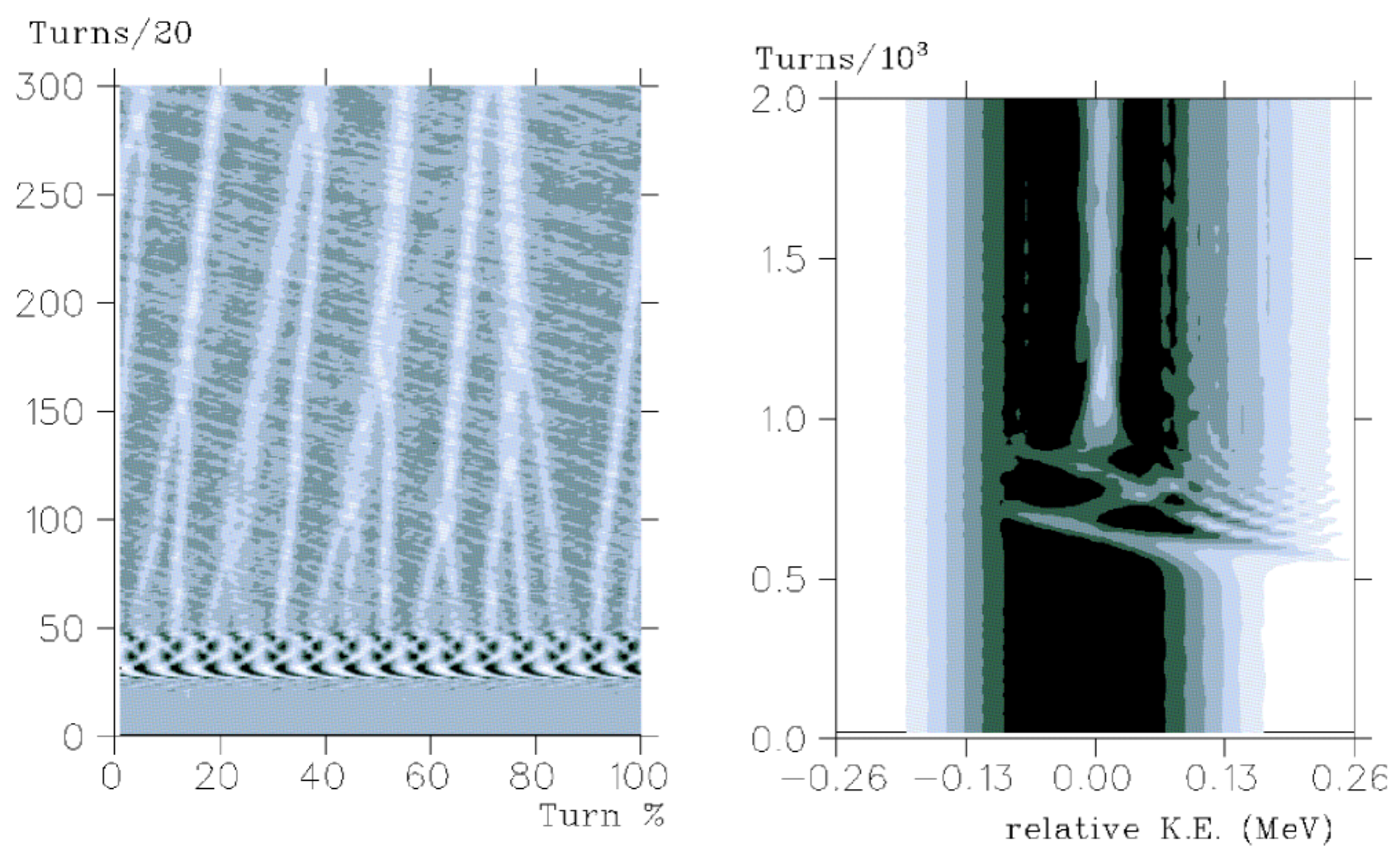

FIG. 18. Waterfall plots of line density (left) and energy spectrum (right) for 6000 and 2000 turns, respectively. $2 \times 10^{12}$ protons, $8 \times 10^{5}$ macroparticles.
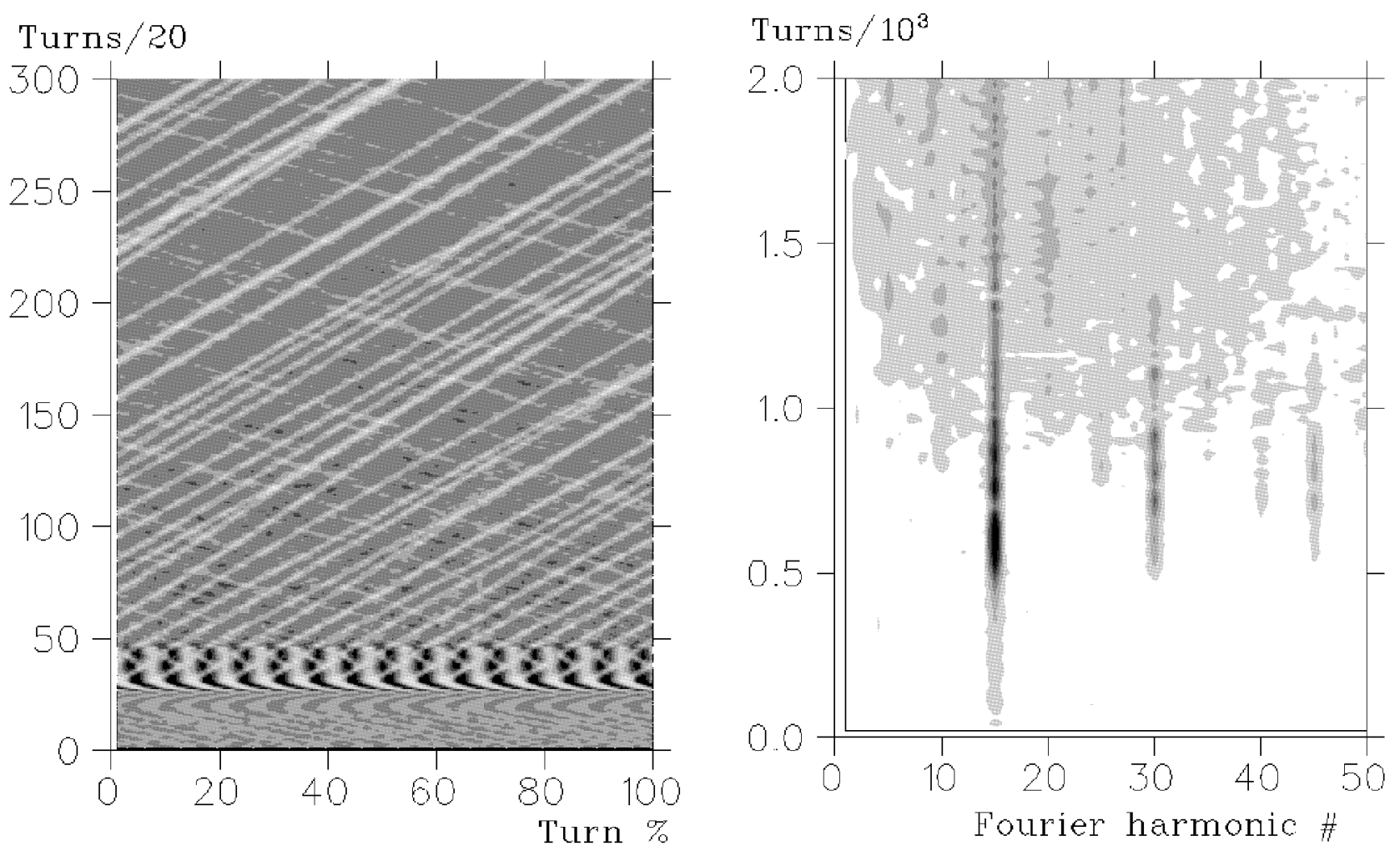

FIG. 19. Waterfall plots of line density (left) and Fourier content (right) for 6000 and 2000 turns, respectively. $4 \times 10^{12}$ protons, $10^{6}$ macroparticles. 


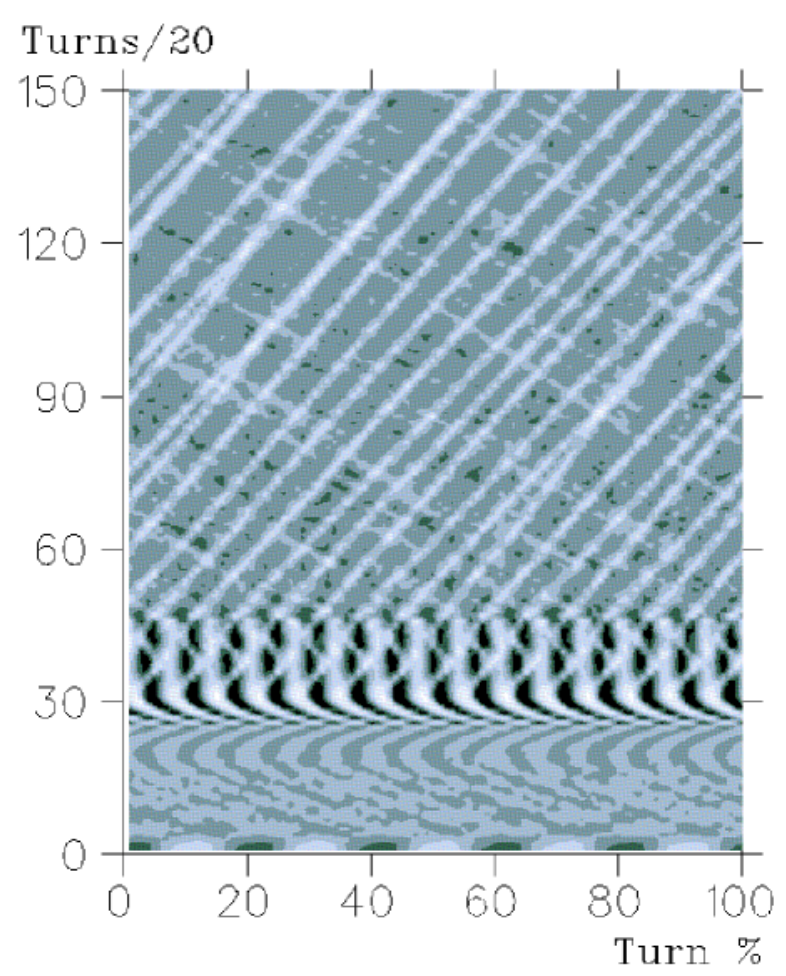

FIG. 20. Waterfall plot of line density, 2400 turns, $4 \times 10^{12}$ protons. Beam has artificial initial $\pm 5 \%$ density modulation which very quickly debunches.

content of the holes clearly survives for more than 2000 turns.

\section{Effect of density modulation}

To test the hypothesis that it is momentum differences that steer the holes and not forces between them, a special ensemble was prepared and the rf-bucket-deposit simulations were repeated. An artificial one-dimensional density modulation was added to the initial ensemble according to $\psi_{0}=\left(1-p^{2}\right)^{2}(1-\alpha \cos 5 x)$, where the azimuth $|x| \leq \pi$ and $\alpha>0$ was varied from $1 \%$ to $10 \%$. In all cases there was no discernible correlation with the hole trajectories, and, indeed, the initial modulation typically debunched well before the bucket deposit was completed, as in Fig. 20.

\section{B. Simulation of rectangular holes}

In order to test the simple theoretical model of Sec. III and to gain an understanding of the phase-space dynamics under simpler circumstances, computer simulations were made with one or more bitrapezoidal holes in phase space. To comply with the model, the holes were cut (down to zero particle density) from a background quasiparabolic distribution of substantially larger momentum width. However, because the space-charge wake is truncated to 50 harmonics and infinitely sharp edges cannot be represented, the holes were made bitrapezoidal.

\section{Single hole}

Figures 21 and 22 show the case of a single hole prepared with parameters that should give a stationary perturbation according to Eq. (7). There is a slight oscillation of the hole's length, which can be attributed to the Fourier truncation of the wake, but it is basically matched in confirmation of the stationarity condition.

The striations appearing in the phase-space scatter plots are due to the fact that the ensemble was initially prepared in colored bands for the purpose of emphasizing and visualizing the particle flows.

Simulations were repeated for the cases of beam current one-half and double the value required for stationarity. In the former case, the hole debunches because the shearing due to momentum spread overcomes the space-charge focusing. In the latter case, Figs. 23-25, the hole quickly breaks up into two smaller holes which thereafter appear almost stationary. The momentum spread of the holes is unaltered, but the charge in each is halved and so the condition for stationarity is restored.
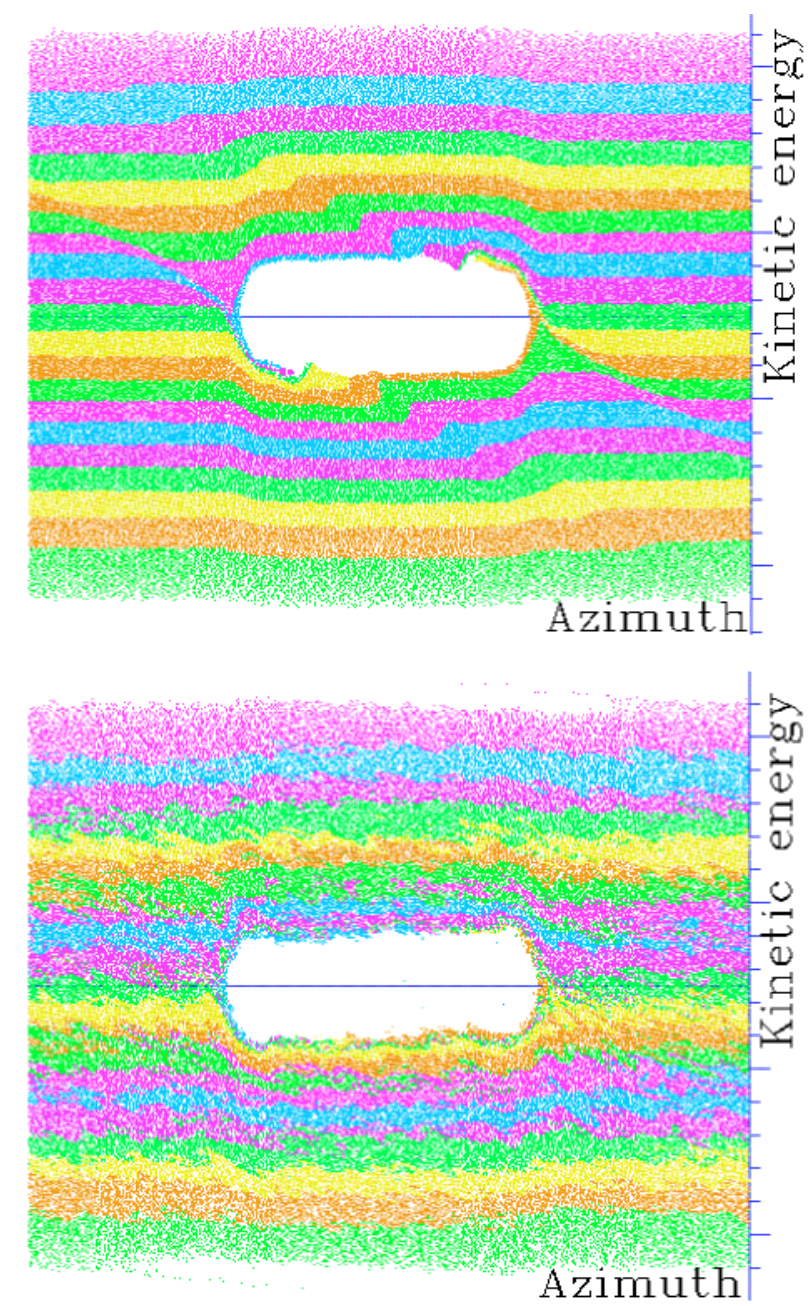

FIG. 21. (Color) Phase space scatter plots at $-14.74 \mathrm{~ms}$ (top) and $-12.49 \mathrm{~ms}$ (bottom), $5 \times 10^{12}$ protons. 


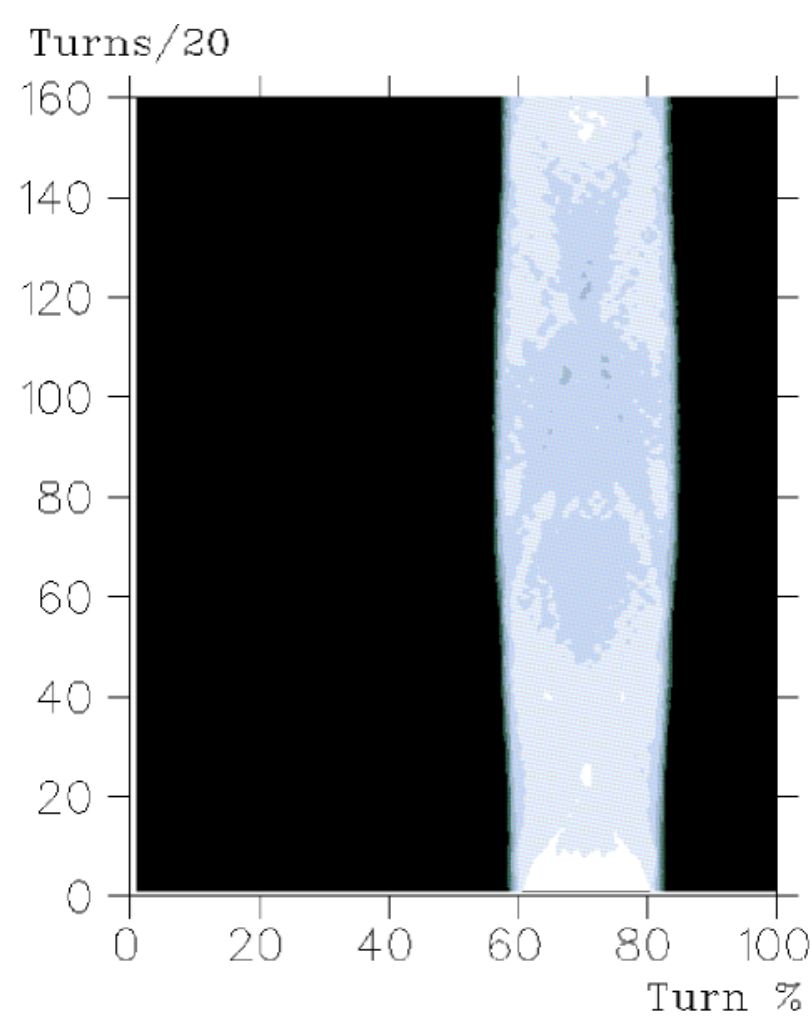

FIG. 22. Waterfall plot of line density for single matched hole (but offset in azimuth) for 3200 turns. Beam current set to give stationary hole. $5 \times 10^{12}$ protons, $2.4 \times 10^{5}$ macroparticles.

\section{Collision of two holes}

Phase-space distributions were prepared with two rectangular holes of slightly different central momentum and initially separated in azimuth. Trials were made in which only the intensity varied. Because of the momentum difference the holes move toward one another and collide in phase space.

The case of two initially matched holes is shown in Figs. 26 and 27: the holes encounter and slide past one another; afterward there is little deformation of their shapes.
For one-half the matched beam current, Figs. 28-30 the holes begin to debunch, then collide and slide past one another, and then continue to debunch indefinitely; $2.2 \times 10^{5}$ macroparticles were employed in the simulation.

For double the matched beam current, Figs. 31-33 each hole begins to divide in two as they collide, the nascent hole pairs slide past one another and continue to divide. When the breakup is complete, the two pairs of holes have become interleaved, being tagged by their different initial momenta. The four holes are matched and persist the length of the simulation.

\section{SOLUTIONS OF THE VLASOV EQUATION}

We now examine the question of whether stationarity conditions such as (3) can be obtained from solution of the time-independent Vlasov equation.

Let $p$ and $q$ be canonical momentum and position coordinates, respectively, and $H$ the Hamiltonian. We assume coordinates in which the beam centroid appears at rest. Let us employ the dot notation for time derivatives. The evolution of the distribution function $\psi$ for a collisionless plasma is governed by the Vlasov equation,

$$
\begin{aligned}
& {\left[\frac{\partial}{\partial t}+\dot{p} \frac{\partial}{\partial p}+\dot{q} \frac{\partial}{\partial q}\right] \psi=0} \\
& \text { where } \dot{p}=-\frac{\partial H}{\partial q}, \quad \dot{q}=+\frac{\partial H}{\partial p} .
\end{aligned}
$$

To stipulate a stationary solution, one simply sets the partial time derivative to zero.

\section{A. With/without wakefield}

In the wake-free case $\dot{q}=p$ and $\dot{p}=0, \psi(p)$ an arbitrary function is a stationary solution.

In the wakefield case $\dot{p}=f(\psi)$, where $f$ depends on the nature of the wake, and $H=p^{2} / 2+\int^{q} \dot{p} d q$ then
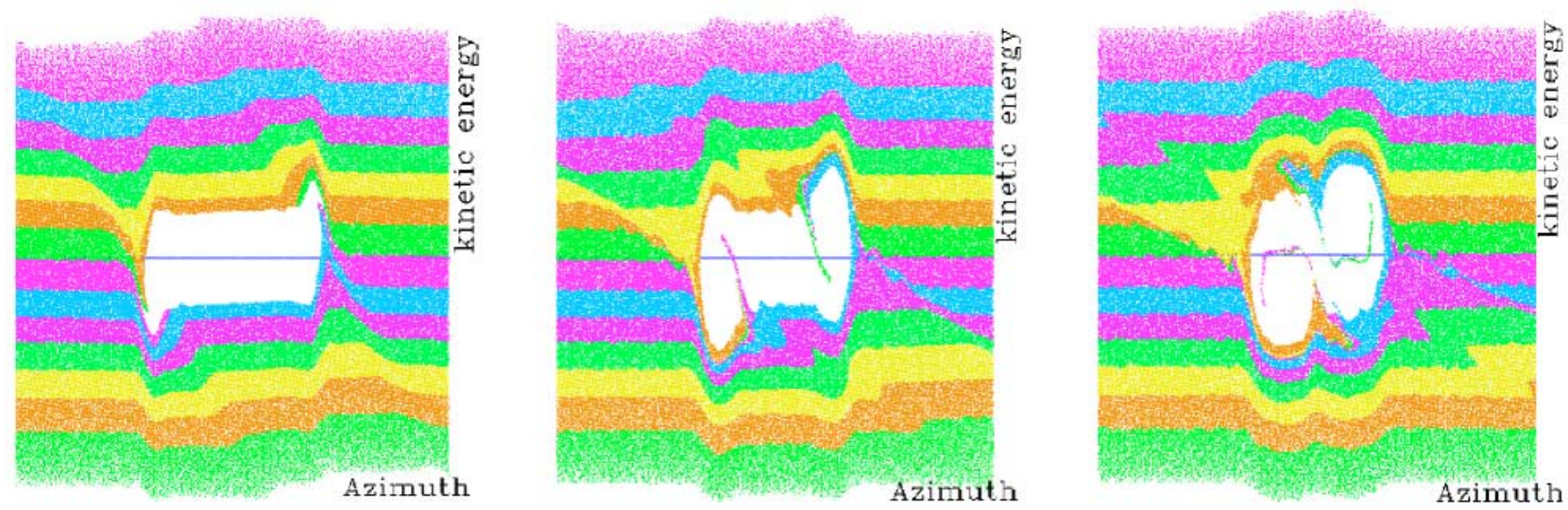

FIG. 23. (Color) Phase space scatter plots (left to right) at $-14.89,-14.74$, and $-14.59 \mathrm{~ms}$, respectively. $10^{13}$ protons, $2.8 \times 10^{5}$ macroparticles. 

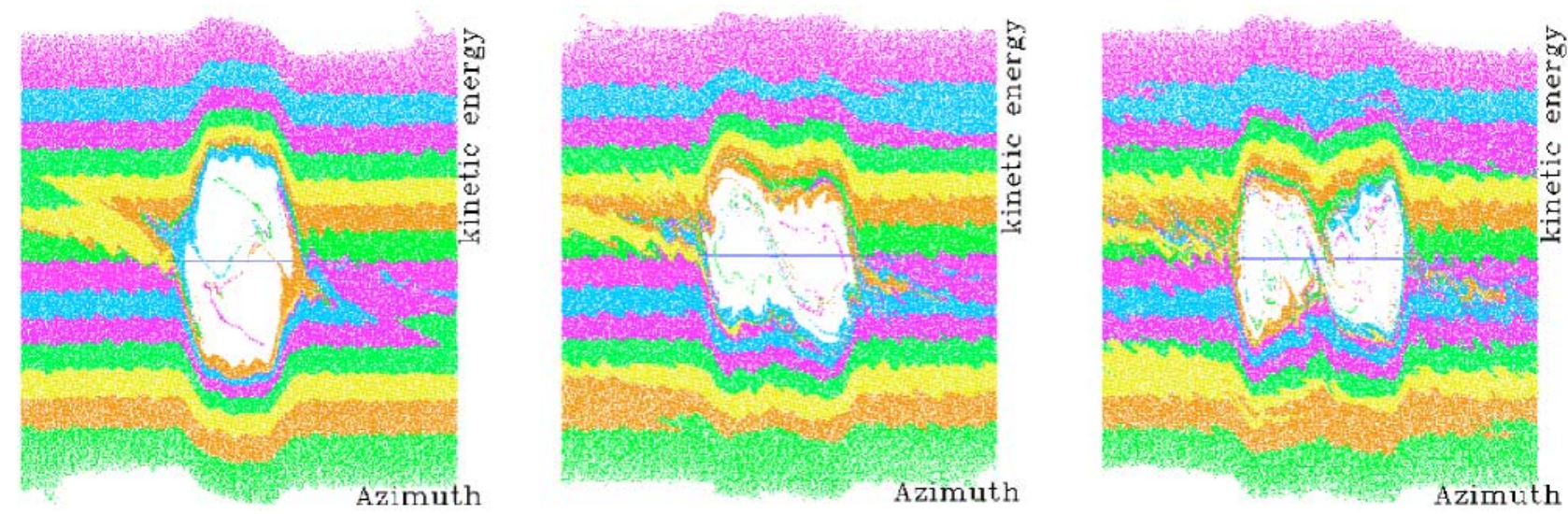

FIG. 24. (Color) Phase space scatter plots (left to right) at $-14.39,-14.09$, and -13.79 ms, respectively. $10^{13}$ protons, $2.8 \times 10^{5}$ macroparticles.

$\psi(H)$ is automatically a stationary solution provided that "self-consistency" is satisfied.

\section{B. Expansion about steady state}

Let $\psi_{0}$ be a steady-state density function and $\psi_{1}$ some perturbation. Substitution of $\psi=\psi_{0}(p)+\psi_{1}(p, q, t)$ into the Vlasov equation yields:

$$
\begin{aligned}
\dot{p} \frac{\partial}{\partial p}\left[\psi_{0}+\psi_{1}\right]+\left[p \frac{\partial}{\partial q}+\frac{\partial}{\partial t}\right] \psi_{1} & =0 \\
\text { where } \dot{p} & =f\left(\psi_{1}\right) .
\end{aligned}
$$

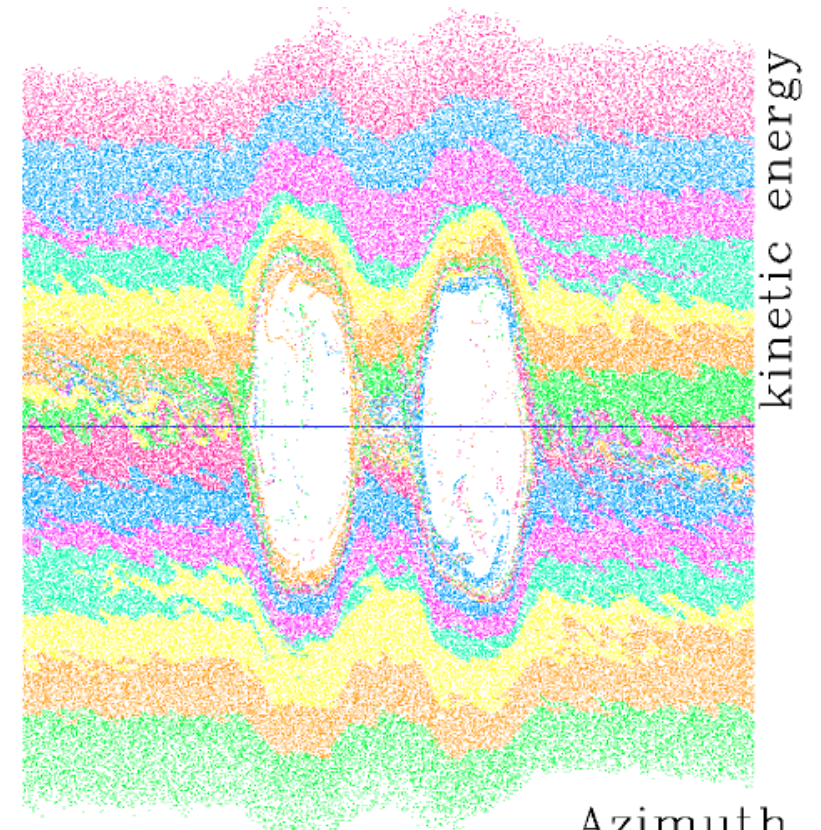

FIG. 25. (Color) Scatter plot at $-13.54 \mathrm{~ms}, 10^{13}$ protons.

\section{Keil-Schnell-type condition}

A perturbation consisting of the superposition of plane waves has the advantage that the net charge is zero, and so there is no need to renormalize the steady-state function. However, to form a localized perturbation a group of waves is required, and the usual Keil-Schnell formalism which deals with single waves and ignores mode coupling may not be adequate. Fortunately, for the case of pure reactive impedance it is possible, for an arbitrary shape perturbation, to derive the dispersion relation for zero modulation frequency more directly and avoid the "wave machinery."

The Vlasov equation (9) is linearized and the time derivative set to zero, leading to

$$
\dot{p}\left(\psi_{1}\right) \frac{\partial \psi_{0}}{\partial p}+p \frac{\partial \psi_{1}}{\partial q}=0 \quad \text { with } \int \psi_{0} d p d q=N .
$$

Here $N$ is the total number of particles in the beam. Let $Z$ represent the coupling between the wake-induced acceleration and the particle number density per unit $q$, denoted $\lambda_{1}(q)=\int \psi_{1} d p$. Thus, for space charge,

$$
\frac{d p}{d t}=-Z \frac{\partial}{\partial q} \int_{-\infty}^{+\infty} \psi_{1}(p, q) d p
$$

Equation (11) is substituted in Eq. (10), one divides throughout by $p$ and then integrates over the momentum coordinate. Then one may cancel $(\partial / \partial q) \int \psi_{1} d p$ as a common factor, leading immediately to the dispersion relation (for zero modulation frequency)

$$
Z \int_{-\infty}^{+\infty} \frac{1}{p} \frac{\partial \psi_{0}}{\partial p} d p=+1
$$

This direct approach could not be used for arbitrary impedance. In general [26], the type of integral appearing in (12) gives rise to a Cauchy principal value (PV) and an imaginary residue. However, under the assumption that $d \psi_{0} / d p=0$ at the beam center $(p=0)$ the residue term is zero. 

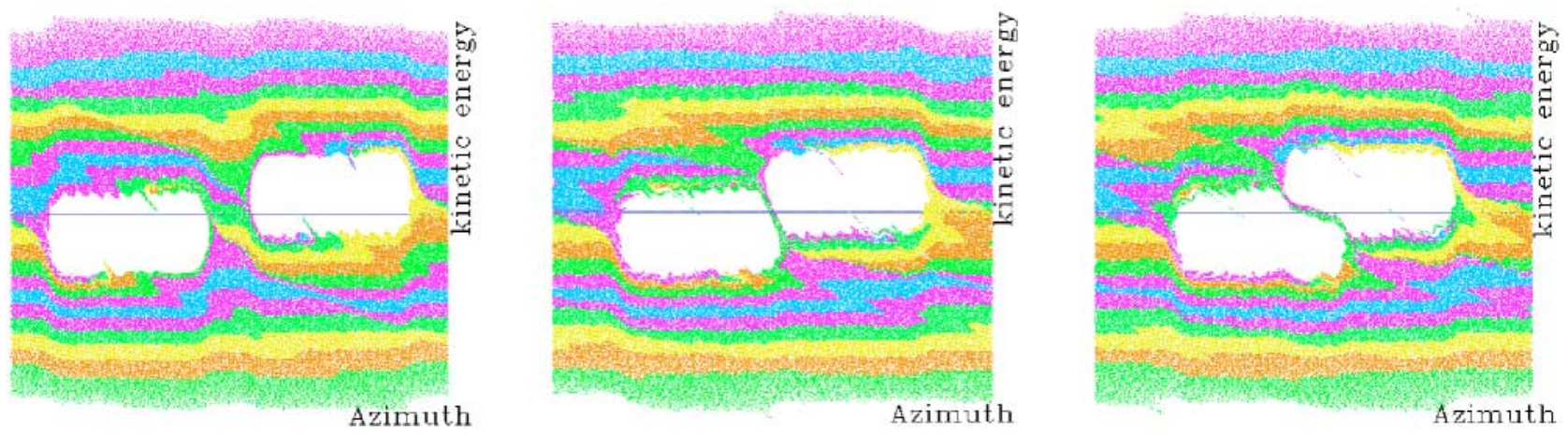

FIG. 26. (Color) Phase space scatter plots (left to right) at $-14.62,-14.37$, and -14.24 ms, respectively. $5 \times 10^{12}$ protons, $2.2 \times 10^{5}$ macroparticles.
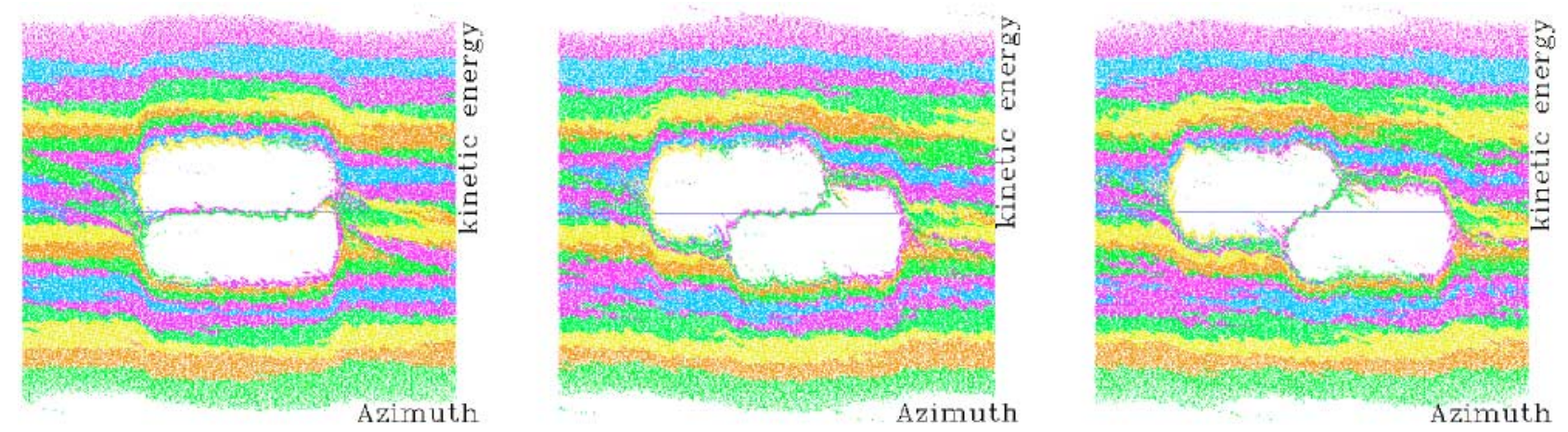

FIG. 27. (Color) Phase space scatter plots (left to right) at $-13.87,-13.62$, and $-13.49 \mathrm{~ms}$, respectively. $5 \times 10^{12}$ protons, $2.2 \times 10^{5}$ macroparticles.

The PV integral is negative/positive if $\psi_{0}$ is locally a maximum/minimum at $p=0$. Thus, as is well known, for capacitive impedance $(Z>0)$, an inverted bell $\psi_{0}$ is required to perpetuate a perturbation below transition energy. Alternatively, if the impedance is pure induc-

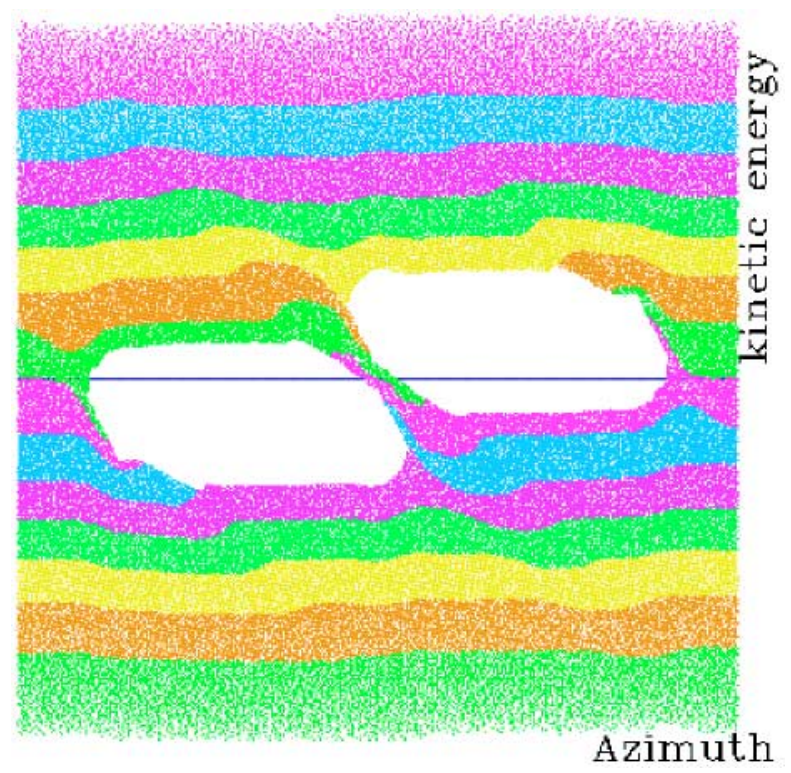

FIG. 28. (Color) Scatter plot at $-14.8 \mathrm{~m}, 2.5 \times 10^{12}$ protons. tive $(Z<0)$ then perturbations may persist for the usual bell-shaped steady-state distributions.

Despite this prediction, the experimentally observed stable holes in the PSB debunched beam occur (below transition energy) for a capacitive impedance (space-charge

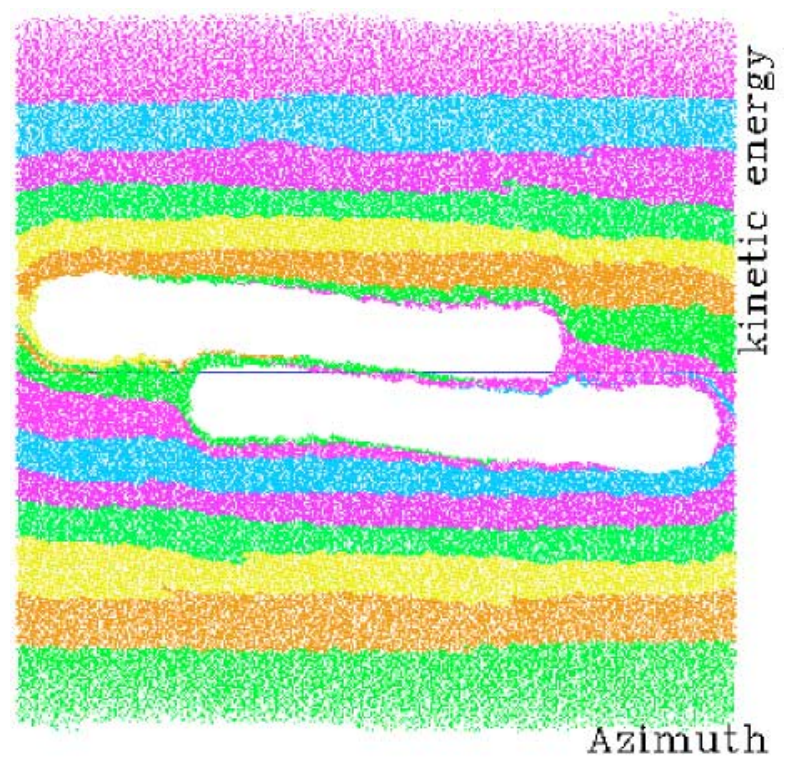

FIG. 29. (Color) Scatter plot at $-13.8 \mathrm{~ms}, 2.5 \times 10^{12}$ protons. 


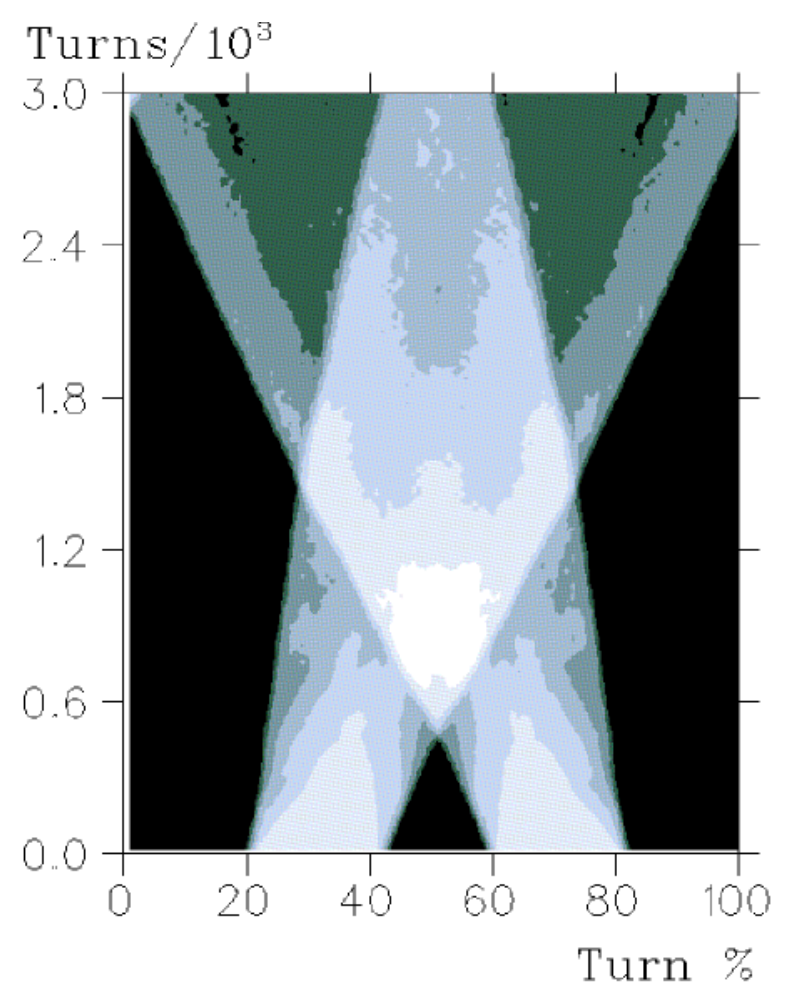

FIG. 30. Waterfall plot of line density, 3000 turns. Two holes debunch, $2.5 \times 10^{12}$ protons, $2.2 \times 10^{5}$ macroparticles.

and perfectly conducting wall) and a quasiparabolic steady-state distribution with $\int(1 / p)\left(\partial \psi_{0} / \partial p\right) d p<0$.

There is another problem with this theory: the ability to cancel the $\lambda_{1}(q)=\int \psi_{1} d p$ terms in the linearized Vlasov equation implies that the perturbation shape $\lambda_{1}(q)$ is an arbitrary function, allowing either holes or spikes, while only holes are seen experimentally in the PSB.

There is a simple corollary to the preceding results:

$$
\psi_{1}=\frac{Z}{p} \frac{\partial \psi_{0}}{\partial p} \lambda_{1}(q) \quad \text { where } \int_{-\pi}^{+\pi} \lambda_{1} d q=n
$$

and $n$ is the number of particles in the perturbation.

\section{Is $\psi_{0}(p)+\psi_{1}(p, q)$ a stationary solution?}

Substitution of $\psi=\psi_{0}(p)+\psi_{1}(H)$ in (8) leaves the residual

$$
\dot{p} \frac{\partial \psi_{0}}{\partial p}+\frac{\partial \psi_{1}}{\partial t}=0
$$

so this cannot be a stationary solution.

Substitution of $\psi=\psi_{0}(p)+P(p) Q(q)$ in (8) leads to the conditions $P=\psi_{0}=e^{-p^{2} / 2}$ and leaves the residual

$$
\dot{p}(1+Q)=\left(\frac{\partial}{\partial q}+\frac{\partial}{p \partial t}\right) Q .
$$

Solutions with $\partial Q / \partial t=0$ will be rare, if any.

Note that the trial form $\psi_{0}(p)+\psi_{1}(p, q)$ is a poor starting point from which to find stationary solutions of the Vlasov equation.

\section{E. Whether to linearize or not}

When linearizing the Vlasov equation, it is customary to state that the perturbation $\psi_{1}$ is small compared with the steady-state function $\psi_{0}$ and that the product $\dot{p} \psi_{1}$ can be neglected. An often unremarked corollary is that

$$
\psi_{1} \propto \frac{1}{p} \frac{\partial \psi_{0}}{\partial p}
$$

and the characteristic scale of the momentum distributions occurring in $\psi_{1}$ and $\psi_{0}$ are similar.

However, the Vlasov equation is not concerned with the absolute amplitude of functions, but rather throughout it is obsessed with derivatives. Consequently, it is not the relative size of $\psi_{0}$ and $\psi_{1}$ that matters, but rather the relative size of their derivatives. Thus for the case of a very localized perturbation, the derivatives of $\psi_{1}$ may be overwhelmingly large compared with $\partial \psi_{0} / \partial p$. In this case, the perturbation decouples (almost) completely from the steady-state distribution and one may neglect the term $\dot{p} \psi_{0}$; in this case $\psi_{1}$ obeys Eq. (8) and $\psi_{1}(H)$ is an (almost) exact stationary solution of the Vlasov equation provided self-consistency is satisfied. In the case that the
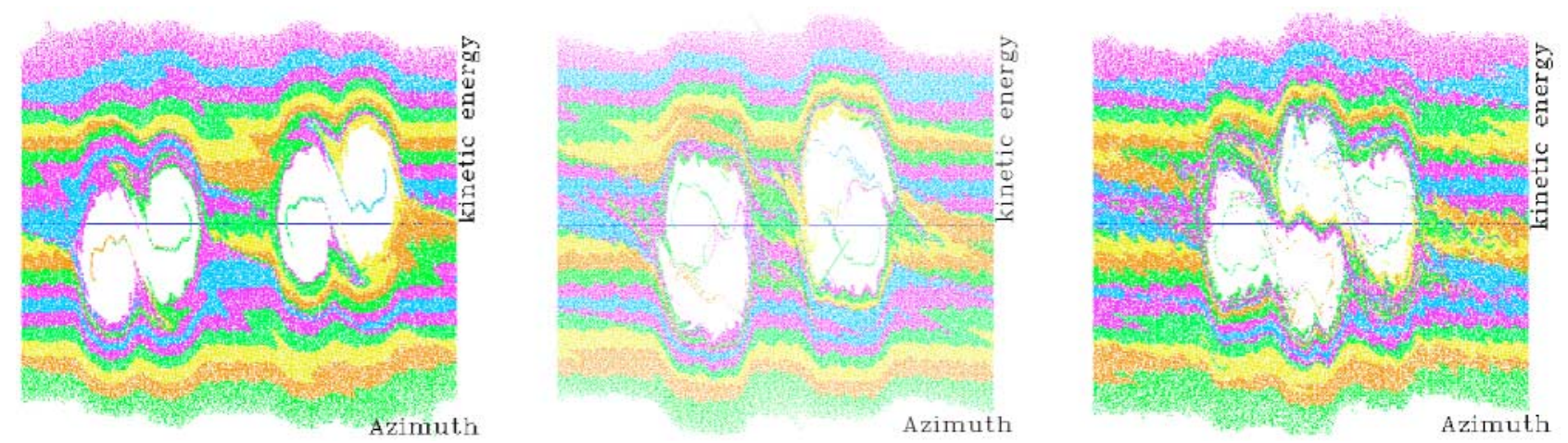

FIG. 31. (Color) Phase space scatter plots (left to right) at $-14.62,-14.37$, and $-14.12 \mathrm{~ms}$, respectively. $10^{13}$ protons, $2.2 \times 10^{5}$ macroparticles. 

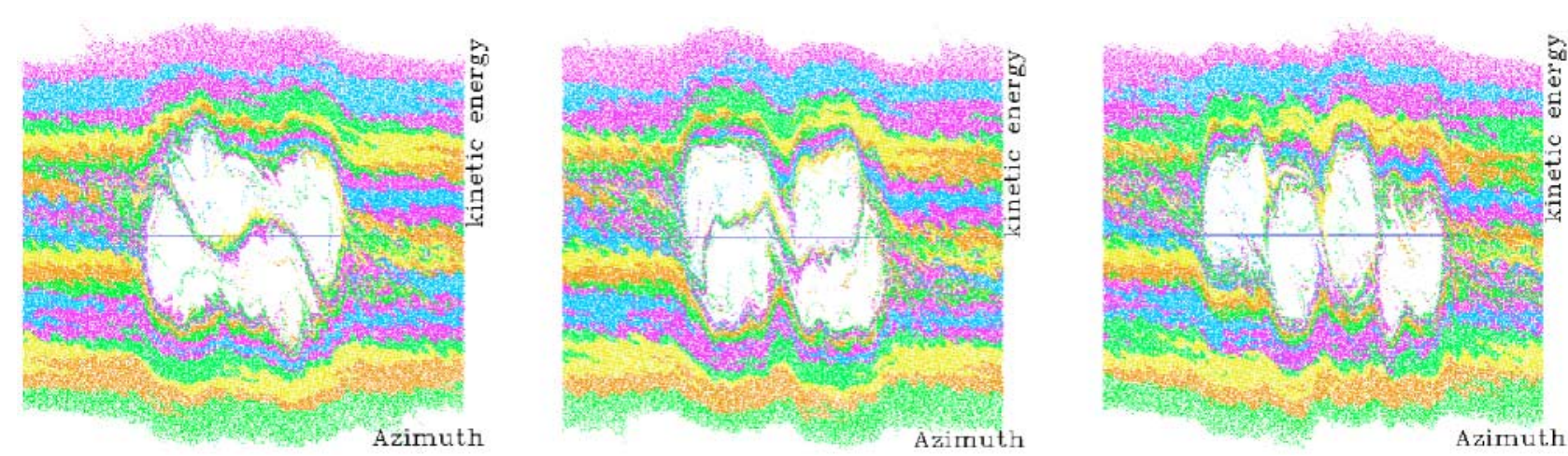

FIG. 32. (Color) Phase space scatter plots (left to right) at $-13.99,-13.87$, and $-13.74 \mathrm{~ms}$, respectively. $10^{13}$ protons, $2.2 \times 10^{5}$ macroparticles.

wake derives from longitudinal space charge, we shall demonstrate explicitly how to construct self-consistent, stationary solutions. We shall consider two types of solutions: $\psi=P(p) Q(q)$ and $\psi(H)$. In both cases the intrinsic nonlinearity of the Vlasov equation is essential to forming self-trapped distributions.

Because the solitonlike $\psi_{1}$ decouples form $\psi_{0}$ there is no need for the momentum center of the perturbation to coincide with that of the beam. Consequently, one may have to make a coordinate transform to the rest frame of the perturbation; we shall assume that this has been done.

\section{F. Dispersion relation}

The stationary $\psi_{1}$ obeys the approximate equation

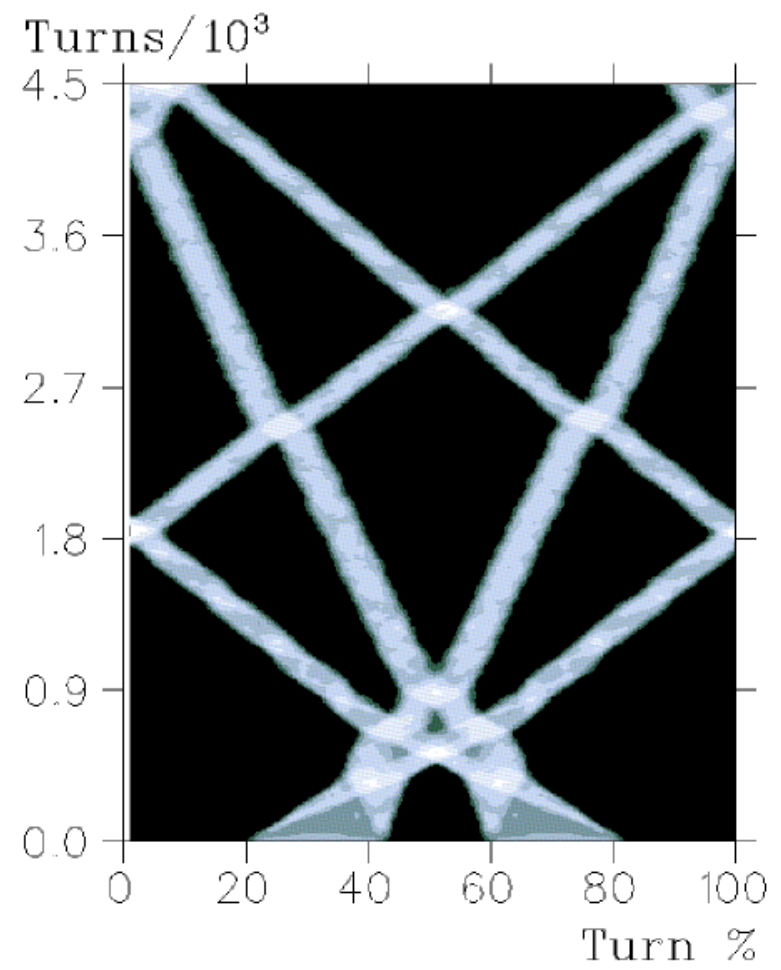

FIG. 33. Waterfall plot of line density, 4500 turns. Two holes split, $10^{13}$ protons, $2.2 \times 10^{5}$ macroparticles.

$$
\dot{p}\left(\psi_{1}\right) \frac{\partial \psi_{1}}{\partial p}+p \frac{\partial \psi_{1}}{\partial q}=0 \quad \text { with } \int \psi_{1} d p d q=n
$$

where the number of particles $n$ in the perturbation is yet to be determined. We substitute (11) in place of $\dot{p}$ and perform the same mathematical manipulations as in Sec. V C to obtain a dispersion relation analogous to (12),

$$
Z \int_{-\infty}^{+\infty} \frac{1}{p} \frac{\partial \psi_{1}}{\partial p} d p=+1
$$

Evidently, $\psi_{1}$ must be an inverted bell shape, that is a hole, for the dispersion relation to be fulfilled (assuming $Z>0$ and capacitive impedance below transition). The dispersion relation (18) is similar to that of Keil-Schnell, but differs in two respects: whereas the latter contains the full beam current (proportional to $N$ ) and the full momentum spread of the beam, the former contains only the current in the hole (proportional to $n$ ) and the momentum width of the hole.

$$
\text { G. } \psi_{1}=P(p) Q(q)
$$

We substitute the trial solution

$$
\psi_{1}=\frac{e^{-p^{2} / 2 \sigma^{2}}}{\sigma \sqrt{2 \pi}} \lambda_{1}(q)
$$

where $\sigma$ is an arbitrary range parameter, into the timeindependent Vlasov equation (17), and we substitute the wake-induced acceleration (11) in place of $\dot{p}$, leading to

$$
Z \frac{\partial \lambda_{1}}{\partial q} \frac{1}{\sigma^{2}} \lambda_{1}(q)+\frac{\partial \lambda_{1}}{\partial q}=0
$$

Cancellation of $\partial \lambda_{1} / \partial q$ throughout leads to the condition that $\lambda_{1}(q)$ is a negative constant,

$$
\lambda_{1}=-\sigma^{2} / Z .
$$

Hence $\lambda$ must be the inverted "top hat" function given by (21) within some range of $|q| \leq \Delta q$ and zero outside that range. A series of equal top hats is also possible. This 
solution is somewhat unphysical but has several properties which are key features of a more realistic model.

The number density (i.e., the amplitude of $\psi_{1}$ ) is negative so that only holes are possible below transition energy. However, particle number density may only be positive (or zero) and so the positive, background, smooth steady-state distribution $\psi_{0}$ is absolutely essential to the physical realization of the perturbation. Thus $\psi_{0} \geq\left|\psi_{1}\right|$ for all $p, q$ is a necessary condition.

The structure is perpetuated by impulses at head and tail which reverse the direction of motion of trapped particles; what happens and what length between head and tail is not terribly important.

The self-consistency condition (21), which derives from the Hamiltonian $H=p^{2} / 2+Z \lambda_{1}$, makes no mention of the length in $q$ of the perturbation. Essentially, this is because we assumed $\psi_{1}$ and $\psi_{0}$ completely decoupled and so lost the property of "number conservation of particles"; a more correct theory would renormalize $\psi_{0}$ and $\psi_{1}$. Our theory is valid for the case that the perturbation does not significantly change the number of particles $N$. Hence the condition $|n|=2 \Delta q \sigma^{2} / Z \ll N$.

Despite the decoupling of $\psi_{0}$ and $\psi_{1}$, the steady-state distribution does impose some constraints on the perturbation. Because the total number density must be positive, the phase-space density $\psi_{0}$ must be high enough to support the perturbation. That is to say, for a hole of given momentum width, there is a critical phase-space density below which $\psi_{0}$ cannot act as a transport medium for solitonlike structures. Let us estimate this critical density.

The density $\psi_{1}$ is of the order of $n /(4 \sigma \Delta q)=$ $-\sigma /(4 Z)$. The density $\psi_{0}$ is of the order of $N /(4 \pi \Delta p)$ where $\Delta p$ is the rms width of $\psi_{0}$. Hence the critical density is

$$
N /(\pi \Delta p) \geq \sigma / Z .
$$

The self-consistency condition (21) implies a very strict relation between the momentum width $\sigma$ of a hole and its depth $\lambda_{1}$, such that progressively narrower holes $(\sigma<1)$ become exceedingly shallow and hard to detect experimentally.

$$
\text { H. } \psi_{1}=\psi_{1}(-H)
$$

A function of the Hamiltonian $H$ is automatically a stationary solution of the Vlasov equation. The Hamiltonian is

$$
H=p^{2} / 2+Z \lambda_{1}(q) \text { with } \lambda_{1} \leq 0 .
$$

The separatrix, which gives the boundary of the perturbation $\psi_{1}$, is given by $H=0$. Within this boundary particles circulate periodically on closed paths, and for this reason Schamel [10] has described the solitonlike solutions as vortices.

The self-consistency condition constrains the form of $\psi_{1}$, and one anticipates the Hofmann-Pedersen [27] form $\psi_{1}=\kappa \sqrt{-H}$ where the constant $\kappa$ is determined by the condition $\lambda_{1}=\int \psi_{1} d p$. The integral is evaluated using the change of variable $p^{2} / 2=-Z \lambda_{1} \sin ^{2} \theta$, yielding $\lambda_{1}=$ $-\kappa Z \lambda_{1} \pi / \sqrt{2}$. Hence the bound-state solution is

$$
\psi_{1}=-\frac{\sqrt{2}}{Z \pi} \sqrt{-p^{2} / 2-Z \lambda_{1}(q)}
$$

where the shape $\lambda_{1}$ is an arbitrary function. This function (24) automatically satisfies the dispersion relation (18). The condition for critical density $\psi_{0} \geq\left|\psi_{1}\right|$ implies

$$
\frac{N}{2 \pi \Delta p} \geq \sqrt{\frac{-\lambda_{1}(q)}{2 Z}} .
$$

The depth of $\lambda_{1}$ and the maximum extent of the boundstate momentum excursions $\pm \hat{p}$ are related by solving $H=0$. Thus we find the density condition

$$
N /(\pi \Delta p) \geq \hat{p} /(2 Z) \text {. }
$$

\section{Pure resistive wake}

For the case of a pure resistive wake

$$
\dot{p}=Y \int \psi_{1} d p=Y \lambda_{1}(q),
$$

where $Y$ is a coupling constant, one may substitute the trial form $\psi_{1}=P(p) Q(q)$ given in Eq. (19) in the timeindependent Vlasov equation. After performing the partial derivative $\partial \psi_{1} / \partial p$, the common factor $p$ may be canceled throughout, then after integrating over momentum $p$ one obtains the equation

$$
\frac{\partial \lambda_{1}}{\partial q}=\frac{Y}{\sigma^{2}} \lambda_{1}^{2}
$$

which has solution

$$
\lambda_{1}(q)=-1 /\left[\kappa+q Y / \sigma^{2}\right],
$$

where $\kappa$ is a constant of integration. Unfortunately, this solution is unphysical, essentially because the lack of coupling to $\psi_{0}$ has destroyed the requirement of finite and positive number density. Equation (29) has both negative and positive infinite number density at the singularity $q=-\kappa \sigma^{2} / Y$, though the function is integrable in the Cauchy principal value sense and the soliton has zero net charge. However, if resistive-wake solitons exist one should expect them to exhibit some type of localized bipolar shape.

\section{J. Keil-Schnell revisited}

Another possible modification to the Keil-Schnell criterion is based on the following observation. Because each plane-wave-type perturbation is equally positive and negative and because a superposition of finite, rather than infinitesimal, wave amplitudes are required to make deep holes, as discussed in Secs. II G and III, one must concede that the steady state distribution $\psi_{0}(p)$ is not arbitrary. If 
one uses plane waves, then it is a mathematical fact that accompanying any deep hole there is also a steep hill at either side. Figure 34 shows a Gaussian $\psi_{0}$, a square-wave perturbation $\psi_{1}$, and their sum gives rise to an unwanted ridge in addition to the desired hole. Hence one must cut a trench into the steady state distribution, along the azimuth, before adding the wave group, as shown in Fig. 35 .

It is worth noting that there is no alternative to the trench type $\psi_{0}$ that is consistent with "cutting a hole from a smooth background distribution." If, as in Fig. 36, one takes a $\psi_{0}$ with a ridge and superposes an inverted squarewave perturbation, the result is a hot spot and not a hole.

For a given hole configuration, there is a precise set of plane waves required and a definite prescription for how to modify the steady-state distribution. Consider a localized perturbation of the form $\psi_{1}=P(p) Q(q)$, where $P \geq 0$ is bell shaped and where $Q=(b-\pi) / \pi$ for azimuth $|q| \leq b<\pi$ and $Q=(b / \pi)$ for azimuth $\pi \geq|q|>$ $b>0$. Further, $P(0)=d$ where $d$ is the depth of the hole. Then the steady state is modified as follows:

$$
\psi_{0}^{\text {new }} \Rightarrow \psi_{0}^{\text {old }}-(b / \pi) P(p)
$$

with the renormalization condition $2 \pi \int \psi_{0}^{\text {new }}=N$. It is assumed that $P(p)$ is known and that $\psi_{0}^{\text {new }}+\psi_{1} \geq 0$ for all $p, q$. In this case one can immediately write down the dispersion relation (for zero modulation frequency)

$$
Z \int_{-\infty}^{+\infty} \frac{1}{p} \frac{\partial}{\partial p}\left[\psi_{0}^{\text {old }}-\frac{b}{\pi} P(p)\right] d p=+1,
$$
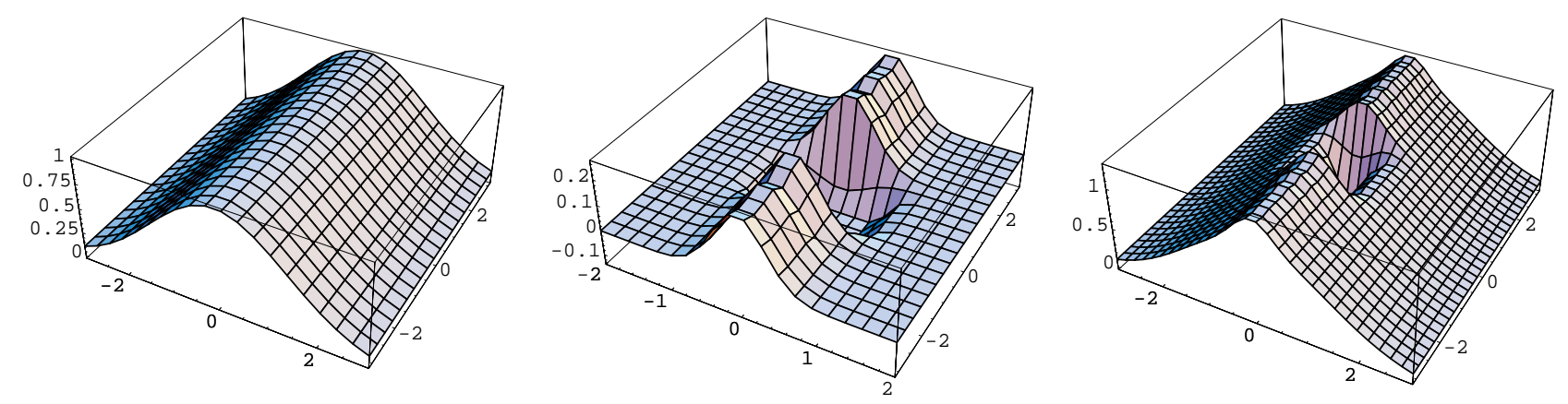

FIG. 34. (Color) Phase space distributions. Left to right: steady-state distribution $\psi_{0}$; square-wave perturbation $\psi_{1} ; \psi_{0}+\psi_{1}$ has a hole and a ridge.
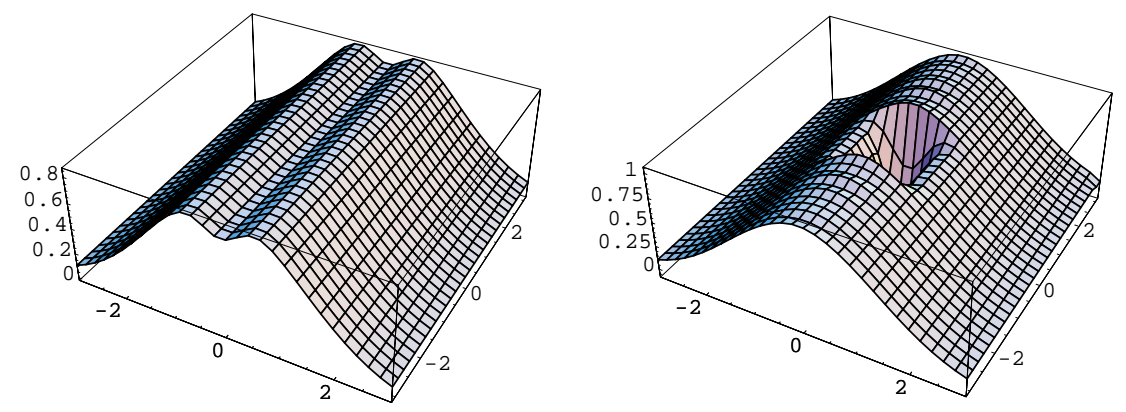

FIG. 35. (Color) Phase space distributions. Left: distribution $\psi_{0}$ with trench. Right: $\psi_{0}+\psi_{1}$ hole but no ridge.
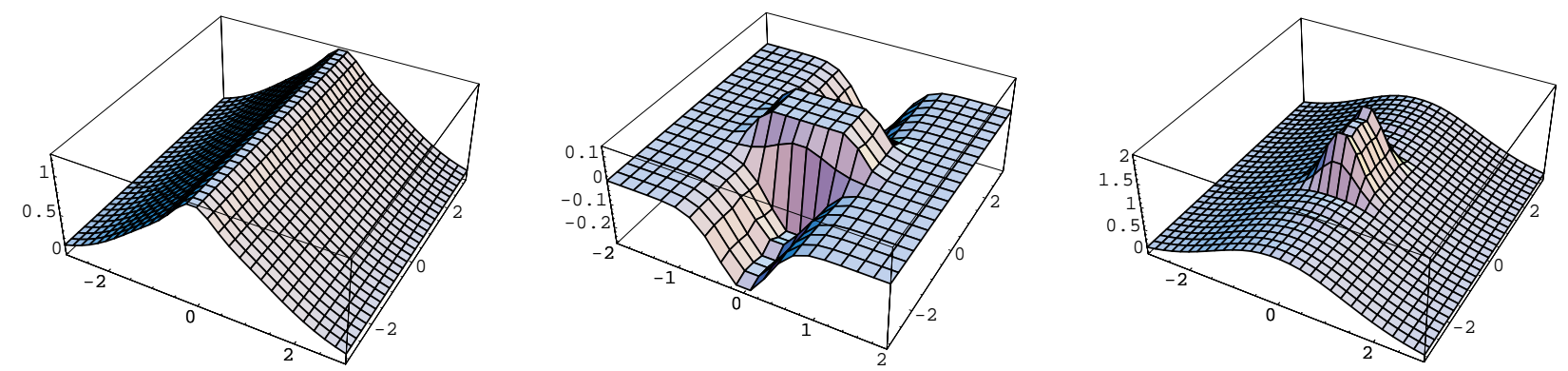

FIG. 36. (Color) Phase space distributions. Left to right: distribution $\psi_{0}$ with ridge; inverted square-wave $\psi_{1} ; \psi_{0}+\psi_{1}$ has a hot spot. 
analogous to the Keil-Schnell condition (12). For sufficiently steep $P(p)$, the modified $\psi_{0}^{\text {new }}$ now allows stationary solutions with capacitive impedance below transition. For example, consider the distribution function

$$
\psi_{0}^{\text {new }}=\frac{N\left[\exp ^{-p^{2} / 2(\Delta p)^{2}}-(b d / \pi) \exp ^{-p^{2} / 2 \sigma^{2}}\right]}{\sqrt{2 \pi}(\Delta p-\sigma b d / \pi)},
$$

whose dispersion integral is

$$
Z N\left[\frac{b d}{\sigma}-\frac{\pi}{\Delta p}\right] /(\Delta p \pi-\sigma b d) .
$$

This quantity is positive for $\sigma / \Delta p<b d / \pi$, and when this condition is satisfied we may expect stationary holes.

This approach is extendable to nonzero modulation frequencies and arbitrary impedance, though it is not fully self-consistent. One expands the perturbation in a set of plane waves $\sum_{k} P_{k}(p) e^{i\left(k q-\omega_{k} t\right)}$, leading to the usual Keil-Schnell dispersion relation for the coherent frequencies $\omega_{k}$, assuming no mode coupling. It is customary to solve this relation by scanning $\omega_{k}$, finding for which value of the impedance is the relation satisfied, and plotting the result in the impedance plane as a "stability diagram." For the double-humped distribution function above [Eq. (32)], the stability diagram is quite complicated: there are regions where for each value of impedance there are two solutions for $\omega_{k}$, which implies there are two possible oscillation modes. When $\sigma / \Delta p>b d / \pi$ there are

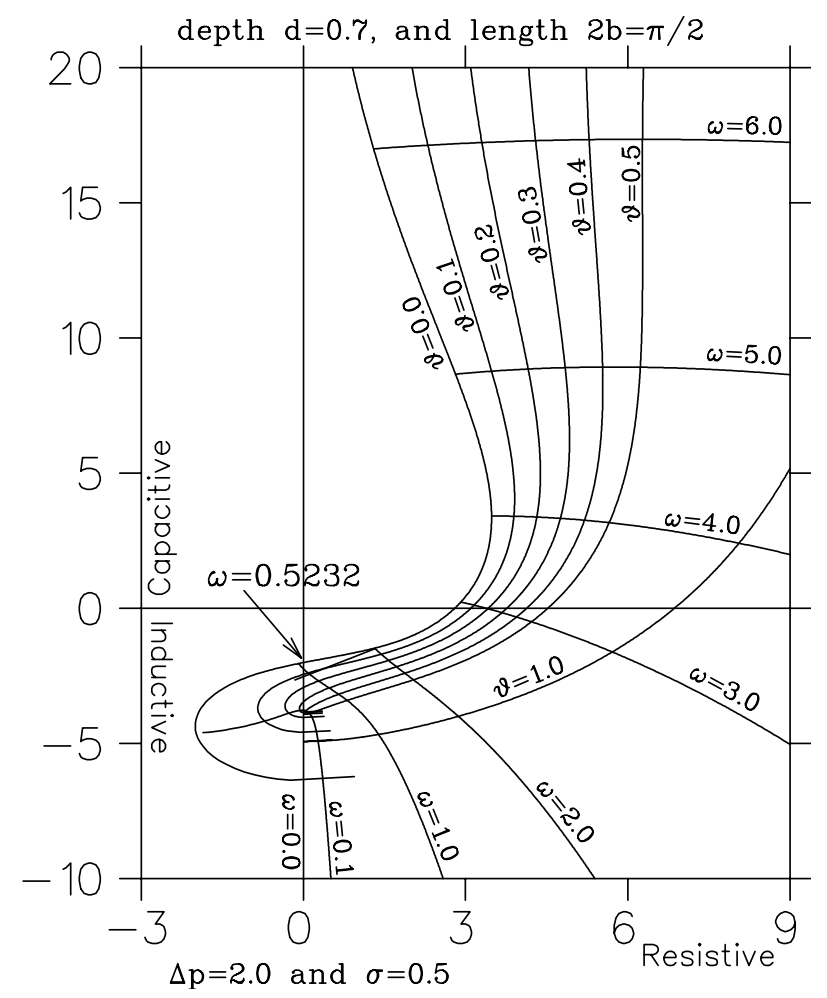

FIG. 37. Stability diagram for beam with wide Gaussian trench $\sigma / \Delta p>b d / \pi(\sigma / \Delta p=0.25)$.

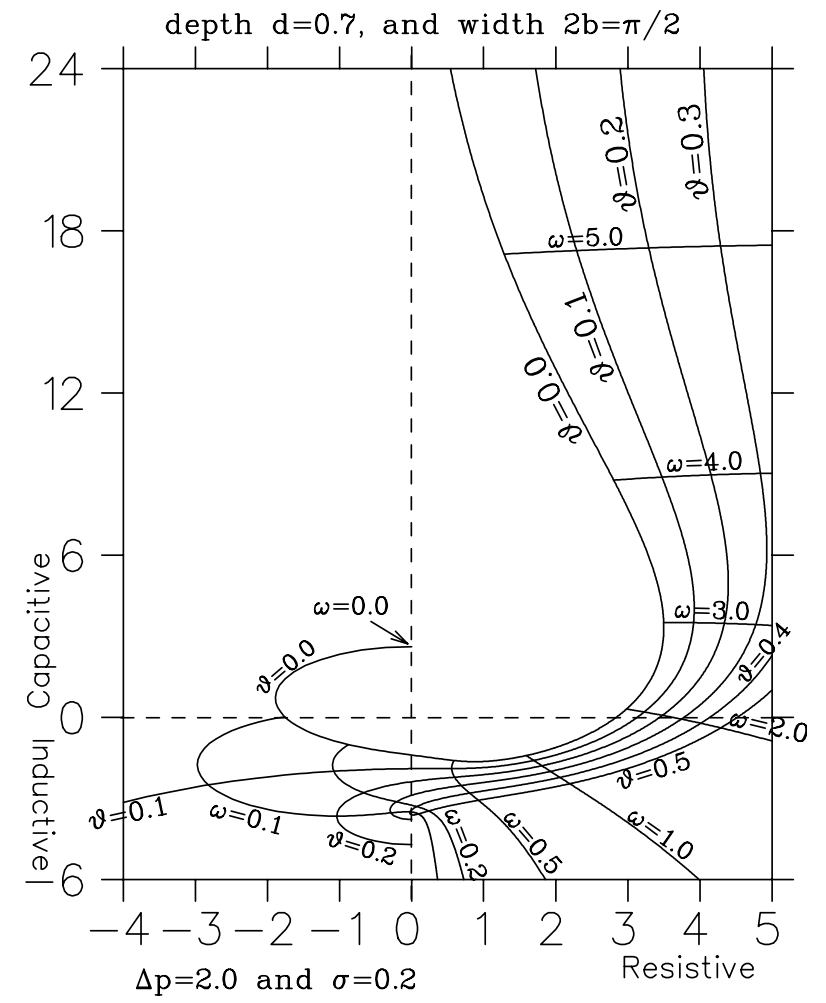

FIG. 38. Stability diagram for beam with narrow Gaussian trench $\sigma / \Delta p<b d / \pi(\sigma / \Delta p=0.10)$.

two modes possible with pure inductive impedance (see Fig. 37), and when $\sigma / \Delta p<b d / \pi$ there is one stationary mode possible with capacitive impedance (see Fig. 38 ). Figures 37 and 38 show the kind of stability diagrams that are to be anticipated as parameters are varied. In these examples $b=\pi / 4, d=0.70$ and $b d / \pi=0.175$. We write the complex frequency as $\omega_{k}=\omega+i \vartheta$. For clarity we show only the branch $0 \leq \omega<+\infty$; the other branch is mirror symmetric about the ordinate. Thus, the stability boundary of Fig. 38 corresponds to the part of the $\vartheta=0$ curve as plotted for negative resistance and its mirror image for positive resistance.

\section{CONCLUSION}

There is much experimental evidence at the CERN PSB to support the existence of near-stationary longitudinal voids. Most evidence suggests that these holes do not coalesce, but that they can break up into smaller holes if the density is high enough. There is strong supporting evidence from computer simulations and theoretical analysis that the holes are perpetuated by space-charge forces. Given these observations, such holes are expected to be ubiquitous in low energy "booster-type" synchrotrons that operate below transition and with space charge as the dominant longitudinal impedance. The behavior of phasespace holes below transition energy is analogous to the behavior of hot spots above transition. However, due to the 
energy dependence of the effect, it is more likely to be seen below transition.

We have derived conditions for stationarity of holes that satisfy the requirement of self-consistency; essentially, the relation between the momentum spread and depth of the holes is given by the Hamiltonian - with the constraint that the phase-space density be high enough to support the solitonlike perturbations which are solutions of the nonlinear Vlasov equation. We have shown that the usual Keil-Schnell [12] criterion does not explain the perpetuation of small voids, but rather they decouple from the steady-state distribution provided that their phase-space gradients are sufficiently great. In this case, the stationarity condition is similar to the Keil-Schnell dispersion relation, but it is the current within and the momentum spread of the hole that are the relevant parameters.

We have given conditions for stationarity but only hinted at calculation/determination of stability. Extending the analysis to the time-dependent nonlinear Vlasov equation may prove difficult, and other methods may be needed. For example, one could take the steady-state barrier-bucketlike solutions and make a perturbation analysis about these using the method of Blaskiewicz [28,29] for true barrier buckets. However, one can remark as follows: If the initial localized solitary perturbations grow, there must come a time when the coupling to the steady-state distribution cannot be ignored, and for space-charge forces below transition this must have a stabilizing effect.

\section{ACKNOWLEDGMENT}

TRIUMF receives Federal funding via a contribution through the National Reserch Council of Canada.

\section{APPENDIX: EQUATIONS OF MOTION}

\section{A. Phase advance}

Let $\beta_{s} c$ be the particle speed and $R_{S}$ the mean radius of the ring. The angular revolution frequency is $\omega_{\text {rev }}=$ $\beta_{s} c / R_{s}$. Let $h$ be the harmonic number of the carrier $\mathrm{rf}$. Let $|q| \leq \pi$ be azimuth and $\phi$ rf phase. By convention, $q=-\phi / h$. Let $\Delta P$ be the momentum deviation from the synchronous value $P_{s}$. The particle phase advances at the rate

$$
\frac{d \phi}{d t}=h \omega_{\mathrm{rev}} \eta_{s} \frac{\Delta P}{P_{s}}
$$

where the slip factor is $\eta_{s}=\left[\alpha_{p}-1 / \gamma_{s}^{2}\right]$ and $\alpha_{p}$ is the momentum compaction factor of the lattice. Below transition, $\eta_{s}<0$.

\section{B. Energy change due to space charge}

Let $z$ be path length along the synchronous orbit, and $\Lambda(z)$ the particle density per unit length, such that $\int_{0}^{2 \pi R_{s}} \Lambda d z=N$. The "classical" formula [15] for longitudinal space-charge electric field (in SI units) is

$$
E_{z}=-\frac{e g_{0}}{4 \pi \epsilon_{0} \gamma^{2}} \frac{\partial}{\partial z} \Lambda(z),
$$

where $\epsilon_{0}$ is the permittivity of free space and $e$ is the elementary charge. The geometric factor $g_{0}$ is conventionally given as $g_{0}=1+2 \ln (b / a)$ but should preferably be taken as $g_{0}=0.5+2 \ln (b / a)$. The rate of energy change due to space-charge force is $d E / d t=\beta c q e E_{z}$. Often, we prefer to work with $\mathrm{rf}$ phase $\phi=z h / R_{s}$ in place of orbit length $z$. The following transformations apply: $\partial / \partial z=$ $\left(h / R_{s}\right) \partial / \partial \phi$ and $\Lambda(z)=\left(h / R_{s}\right) \Lambda(\phi)$. Consequently,

$$
\begin{aligned}
\frac{d E}{d t}=-\frac{e^{2} g_{0} \beta c}{4 \pi \epsilon_{0} \gamma^{2}} \frac{h^{2}}{R_{s}^{2}} & \frac{\partial}{\partial \phi} \Lambda(\phi), \\
& \text { where } \int_{0}^{2 \pi h} \Lambda d \phi=N .
\end{aligned}
$$

\section{Hamiltonian}

If we introduce the function

$$
\mathcal{H}=\frac{h \omega_{\mathrm{rev}} \eta_{s}}{2 P_{s}}(\Delta P)^{2}+\left.\frac{d P}{d E}\right|_{s} \frac{e^{2} g_{0} \beta_{s} c}{4 \pi \epsilon_{0} \gamma^{2}} \frac{h^{2}}{R_{s}^{2}} \Lambda(\phi),
$$

the rates of change are given by the derivatives $\dot{\phi}=$ $+(\partial / \partial \Delta P) \mathcal{H}$ and $\dot{\Delta} P=-(\partial / \partial \phi) \mathcal{H}$. Finally, we make a transformation to the coordinates azimuth $q$ and momentum like $p^{2}=\left(-\omega_{\text {rev }} \eta_{s} / P_{s}\right) \Delta P^{2}$ and introduce the Hamiltonian $H=p^{2} / 2+Z \lambda(q)$, where the coupling constant

$$
Z=\frac{e^{2} g_{0}}{4 \pi \epsilon_{0} \gamma^{2} R_{s}^{2}} \quad \text { and } \quad \lambda(q)=\frac{1}{h} \Lambda(\phi) .
$$

[1] P. Colestock and L. Spentzouris, The Future of Accelerator Physics: The Tamura Symposium, AIP Conf. Proc. No. 356 (AIP, New York, 1996).

[2] L. K. Spentzouris, J.-F. Ostiguy, and P. L. Colestock, Phys. Rev. Lett. 76, 620 (1996).

[3] A. Blas et al., CERN PS/RF/Note No. 2000-016 (MD), 1999.

[4] A. Blas et al., CERN PS/RF/Note No. 2000-015 (MD), 1999.

[5] A. Blas et al., CERN PS/RF/Note No. 2000-014 (MD), 1999.

[6] M. Sjöström, Royal Institute of Technology, Sweden, Report No. ISRN KTH/SB/R-97/39-SE, 1997.

[7] F. Sacherer and F. Pedersen (private communication).

[8] H. Schamel, Phys. Rep. 140, 161-191 (1986).

[9] H. Schamel, Phys. Rev. Lett. 79, 2811-2814 (1997).

[10] H. Schamel, Phys. Scr. T75, 23-27 (1998).

[11] H. Schamel, in Proceedings of the 7th European Particle Accelerator Conference, Vienna, 2000, http://accelconf. web.cern.ch/AccelConf/e00/index.html 
[12] E. Keil and W. Schnell, CERN Report No. ISR-THRF/69-48, 1969.

[13] J. Bisognano, in Proceedings of the 5th European Particle Accelerator Conference, Sitges, 1996 (Institute of Physics, Bristol, UK, 1996), p. 328.

[14] S. I. Tzenov, in Workshop on Instabilities of High Intensity Hadron Beams in Rings, edited by T. Roser and S. Y. Zhang, AIP Conf. Proc. No. 496 (AIP, New York, 1999), p. 351 .

[15] A. Hofmann, in Proceedings of Theoretical Aspects of the Behaviour of Beams in Accelerators and Storage Rings, Erice, Sicily (CERN Report No. 77-13, 1977), pp. 140-144.

[16] A. Ruggiero and V. Vaccaro, CERN Report No. ISR/TH/ 68-33, 1968.

[17] J.-L. Laclare, CERN Report No. CERN 85-19, 1985, pp. 377-414.

[18] 9th ICFA Mini-Workshop on High Intensity, High Brightness Hadron Beams, Switzerland, 2000, http://psdata.web. cern.ch/psdata/www/icfa9/ICFAWelcome.html

[19] S. Wolfram, MATHEMATICA, A System for Doing Mathemat- ics by Computer (Addison-Wesley, Reading, MA, 1993).

[20] A. Blas et al., in Proceedings of the 1999 Particle Accelerator Conference, New York (IEEE, Piscataway, NJ, 1999), p. 143.

[21] A. Blas et al., in Proceedings of the 7th European Particle Accelerator Conference, Vienna, 2000 (Ref. [11]).

[22] See S. Hancock, MATHEMATICA notebook psbnotch.nb at http://t.home.cern.ch/t/tomograp/www/

[23] S. Koscielniak, TRIUMF Report No. TRI-DN-97-12, 1997.

[24] S. Koscielniak, in Proceedings of the 1999 Particle Accelerator Conference, New York (Ref. [20]), p. 1839.

[25] http://www.triumf.ca/people/koscielniak/bubbles.htm

[26] L. D. Landau, J. Phys. (Moscow) 10, 25 (1946).

[27] A. Hofmann and F. Pedersen, CERN Report No. CERN/ ISR/TH/79-17, 1979.

[28] M. Blaskiewicz, Phys. Rev. ST Accel. Beams. 1, 044201 (1998).

[29] M. Blaskiewicz, in Proceedings of the 1999 Particle Accelerator Conference, New York (Ref. [20]), p. 1611. 